\title{
DECOMPOSABLE MODULES
}

\author{
BY \\ EBEN MATLIS
}

1. Introduction. The concept of an $h$-local domain was introduced in $[8, \S 8]$ as a simultaneous generalization of both a Dedekind domain and a local domain. It is a domain in which localizations with respect to maximal ideals behave properly. In this paper we give some further characterizations of an $h$-local domain and investigate its ideal structure. The tools found here, together with the theory of cotorsion modules of [8], are then used to settle some questions concerning decomposability of modules that have descended to us from the theory of Abelian groups. The point here is that the machinery of cotorsion modules and $h$-local domains is useful in solving problems that have a classical history, and were posed long before the development of this machinery.

In $\$ 5$ we characterize those $h$-local domains that have the property that every finitely generated torsion module is a direct sum of cyclic modules. This generalizes and proves the converse of [3, Theorem 14]. In $\$ 6$ we find the class of all integral domains having the property that every torsion-free module of finite rank is a direct sum of modules of rank one. This generalizes and proves the converse of [3, Theorem 12]. Finally, in $\S 7$ we find the class of all Noetherian domains having the property that every homomorphic image of the quotient field is indecomposable. This answers a question raised in [7, p. 573].

2. The ideal structure of an $h$-local domain. Throughout this paper $R$ will denote an integral domain (not a field), $Q$ its quotient field, and $K$ the $R$-module $Q / R$. We will let $H$ denote the ring $\operatorname{Hom}_{R}(K, K)$. $H$ is the completion of $R$ in the $R$-topology [8, Proposition 6.4]. $R$ is said to be an $h$-local domain if the following two conditions are satisfied:

(1) Each nonzero ideal of $R$ is contained in only finitely many maximal ideals of $R$;

(2) Each nonzero prime ideal of $R$ is contained in only one maximal ideal of $R$. $R$ is said to be $h$-semilocal if it satisfies merely condition (1).

These types of rings can be characterized by finiteness conditions on the topology of $\Omega_{P}$, the space of prime ideals of $R$; or of $\Omega$, the space of maximal ideals of $R$. A set in $\Omega_{P}$ is called closed if it consists of all of the prime ideals of $R$ containing a given ideal of $R$. A similar statement is true for $\Omega$. It is then easy to see that $R$ is $h$-local if and only if every proper closed set in $\Omega_{P}$ is a finite union of isolated closed sets (a closed set is called isolated, if it contains only one closed point).

Received by the editors March 1, 1965. 
It is also obvious that $R$ is $h$-semilocal if and only if $\Omega$ has the cofinite topology (i.e., the open sets of $\Omega$ are the complements of finite sets).

Examples of $h$-local domains are the following:

(a) Local domains (domains with only one maximal ideal, and no chain conditions assumed).

(b) Dedekind rings.

(c) Noetherian domains of Krull dimension one.

(d) Domains in which every nonzero prime ideal is maximal, and no maximal ideal is contained in the union of the remaining ones (see [8, Proposition 8.7]).

In [8, Theorem 8.5] we proved the following characterization of $h$-local domains.

THEOREM. The following statements are equivalent:

(1) $R$ is an h-local domain.

(2) $S(M) \otimes_{R} R_{M} \cong Q$ for all $M \in \Omega$, where $S(M)=\bigcap R_{N}$ for all $N \in \Omega-\{M\}$.

(3) $K \cong \sum \oplus K_{M}$, where $M$ ranges over all maximal ideals in $\Omega$.

(4) $H \cong \prod H(M)$, where $M$ ranges over all maximal ideals in $\Omega$, and where $H(M)=\operatorname{Hom}_{R_{M}}\left(K_{M}, K_{M}\right)$ is the completion of $R_{M}$.

Definition. An ideal $I$ of $R$ will be called colocal if it is contained in only one maximal ideal $M$ of $R$. $I$ will then be said to belong to $M$. An $R$-module will be called local if it is isomorphic to $R / I$, where $I$ is a colocal ideal of $R$. We have the following elementary properties of colocal ideals;

(1) If $I_{1}, \ldots, I_{n}$ are colocal ideals belonging to $M$, then $I_{1} \cap \ldots \cap I_{n}$ is a colocal ideal belonging to $M$.

(2) If $I$ and $J$ are colocal ideals belonging to different maximal ideals, then $I$ and $J$ are relatively prime; i.e., $I+J=R$.

(3) Let $I=\bigcap_{j=1}^{n} I_{j}$, where each $I_{j}$ is a colocal ideal belonging to a maximal ideal $M_{j}$, and the $M_{j}$ 's are all distinct. Then $I=I_{1} I_{2} \cdots I_{n}$ and $R / I \cong R / I_{1} \oplus \cdots \oplus R / I_{n}$.

(4) If $A$ is a local $R$-module, and if $B$ and $C$ are submodules of $A$ such that $B+C=A$, then either $B=A$ or $C=A$.

Lemma 2.1. Let $I$ be a colocal ideal of $R$ belonging to a maximal ideal $M$. Then:

(1) $R_{M} \otimes_{R} R / I \cong R / I$, and $I_{M} \cap R=I$.

(2) If $N$ is a maximal ideal different from $M$, then $R_{N} \otimes_{R} R / I=0$.

Proof. Let $J=\{r \in R \mid$ there is an $s \in R-M$ such that $s r \in I\}$. Then $J=I_{M} \cap R$, and $I \subset J$. Hence $I_{M}=J_{M}$. Now $1 \notin J$, and so $J$ is a colocal ideal belonging to $M$. Let $L$ be any colocal ideal belonging to $M$, and let $N$ be a maximal ideal different from $M$. Since $L$ is not contained in $N$, it meets $R-N$, and thus $L_{N}=R_{N}$. Hence we have $I_{N}=R_{N}$ and $J_{N}=R_{N}$ for all maximal ideals $N$ different from $M$. But $I_{M}=J_{M}$ also, and thus we have $I=\bigcap I_{N}=\bigcap J_{N}=J$, where the intersection ranges over all maximal ideals $N$ of $R$. Therefore $I_{M} \cap R=I$. 
Define $f: R / I \rightarrow R_{M} \otimes_{R} R / I$ by $f(\bar{x})=1 \otimes \bar{x}$ for all $\bar{x} \in R / I$. If $\bar{x} \in \operatorname{Ker} f$, then there exists an $s \in R-M$ such that $s \bar{x}=0$. Thus if $\bar{x}=x+I, x \in R$, we have $s x \in I$. Hence $x \in J=I$, and $\bar{x}=0$. Therefore, $f$ is 1 to 1 . Let $\bar{y} \in R_{M} \otimes_{R} R / I$; then there exists $\bar{x} \in R / I$ and $s \in R-M$ such that $\bar{y}=(1 / s) \otimes \bar{x}$. Now $R s+I$ can be contained in no maximal ideal of $R$, and so $R s+I=R$. Therefore, $s(R / I)=R / I$, and so there exists an element $\bar{z} \in R / I$ such that $\bar{x}=s \bar{z}$. We then have $\bar{y}=1 \otimes \bar{z}=f(\bar{z})$. Thus $f$ is onto, and so is an isomorphism.

If $N$ is a maximal ideal different from $M$, then we have seen that $I_{N}=R_{N}$, and so $R_{N} \otimes_{R} R / I \cong R_{N} / I_{N}=0$.

Definition. Let $I=\bigcap_{j=1}^{n} I_{j}$ be a finite intersection of ideals, where $I_{j}$ is a colocal ideal belonging to a maximal ideal $M_{j}$. We will say that this decomposition is normal, if $M_{i} \neq M_{k}$ for $i \neq k$. It is clear from elementary property (1) that every finite intersection of colocal ideals can be normalized.

LEMMA 2.2. Let $I=\bigcap_{j=1}^{n} I_{j}$ be a normal decomposition of an ideal $I$, where $I_{j}$ is a colocal ideal belonging to a maximal ideal $M_{j}$. Then $I_{j}=I_{M_{j}} \cap R$, and so the $I_{j}$ 's are unique.

Proof. By Lemma 2.1 we have

$$
I_{M_{k}} \cap R=\left(\bigcap_{j=1}^{n} I_{j}\right)_{M_{k}} \cap R=\bigcap_{j=1}^{n}\left(\left(I_{j}\right)_{M_{k}} \cap R\right)=I_{k} \cap R=I_{k} .
$$

THEOREM 2.3. Let $R$ be an integral domain. Then $R$ is an h-local ring if and only if every nonzero ideal of $R$ is a finite intersection of colocal ideals.

Proof. Suppose that every nonzero ideal of $R$ is a finite intersection of colocal ideals. Let $I$ be a nonzero ideal of $R$. Then $I=\bigcap_{j=1}^{n} I_{j}$, where $I_{j}$ is a colocal ideal belonging to a maximal ideal $M_{j}$. If $M$ is any maximal ideal containing $I$, then $M$ contains one of the $I_{j}$ 's, and hence is equal to that $M_{j}$. Therefore, $I$ is contained in only finitely many maximal ideals of $R$. If $I$ is a prime ideal, then it can not be the intersection of two properly larger ideals, and so it is equal to one of the $I_{j}$ 's. Thus $I$ is contained in only one maximal ideal of $R$. Therefore, $R$ is an $h$-local domain.

Conversely, suppose that $R$ is an $h$-local domain. Let $I$ be a nonzero ideal of $R$; then $I$ is contained in only a finite number of maximal ideals $M_{1}, \ldots, M_{n}$. Let $I_{j}=I_{M_{j}} \cap R$ for $j=1, \ldots, n$. If $N$ is a maximal ideal different from $M_{1}, \ldots, M_{n}$, then $I$ meets $R-N$ and so $I_{N}=R_{N}$. Thus, if we let $M$ range over all maximal ideals of $R$, we have $\bigcap_{j=1}^{n} I_{j}=\bigcap_{j=1}^{n} I_{j} \cap\left(\bigcap R_{M}\right)=\bigcap I_{M}=I$.

It only remains to show that $I_{j}$ is a colocal ideal belonging to $M_{j}$. Since $I \subset M_{j}$, we have $I_{j} \subset M_{j}$. Let $N$ be any maximal ideal different from $M_{j}$. Then $\left(I_{j}\right)_{N}=$ $\left(I_{M_{j}}\right)_{N} \cap R_{N}$. But $R_{M_{j}} \otimes_{R} R_{N} \cong Q$, since $R$ is $h$-local, and so

$$
\left(I_{M_{\mathrm{g}}}\right)_{N}=\left(I \otimes_{R} R_{M_{\mathrm{g}}}\right) \otimes_{R} R_{N} \cong Q
$$


Therefore, $\left(I_{j}\right)_{N}=Q \cap R_{N}=R_{N}$. It follows that $I_{j}$ is not contained in $N$. Hence $I_{j}$ is a colocal ideal belonging to $M_{j}$.

LEMMA 2.4. Let $R$ be an integral domain and I a nonzero projective ideal of $R$. Let $M_{1}, \ldots, M_{n}$ be maximal ideals of $R$. Then there exists an ideal of $R$ isomorphic to $I$ which is not contained in any of $M_{1}, \ldots, M_{n}$; (i.e., there exists an element $q \in I^{-1}$ such that $\left.q I \notin M_{1} \cup \cdots \cup M_{n}\right)$.

Proof. The proof will be by induction on $n$. Suppose that $n=1$. Now $1=\sum_{i=1}^{t} a_{i} q_{i}$, where $a_{i} \in I$ and $q_{i} \in I^{-1}$. Hence we can assume without loss of generality that $a_{1} q_{1} \notin M_{1}$, and so $q_{1} I$ is not contained in $M_{1}$. Suppose that $n>1$, and that the lemma is true for $n-1$. We can assume that the $M_{i}$ 's are all distinct. By the induction hypothesis we may assume that $I$ is not contained in $M_{2} \cup \cdots \cup M_{n}$, but that $I$ is contained in $M_{1}$. Choose $p \in M_{2} M_{3} \ldots M_{n}$ such that $p \notin M_{1}$. By the case $n=1$ there exists an element $q \in I^{-1}$ such that $q I \notin M_{1}$. Let $v=1-p q$; then we can not have $v I \subset M_{i}$ for any $i>1$. For suppose that $v I \subset M_{i}$. Take $x_{i} \in I$ such that $x_{i} \notin M_{i}$; then $x_{i}-p q x_{i}=v x_{i} \in M_{i}$ and $p q x_{i} \in M_{i}$. Thus $x_{i} \in M_{i}$, which is a contradiction. We can not have $v I \subset M_{1}$, either. For take $x \in I$ such that $q x \notin M_{1}$; then $x \in M_{1}$, and if $x-p q x=v x \in M_{1}$, we would have $p q x \in M_{1}$. But $p \notin M_{1}$, and so $q x \in M_{1}$, which is a contradiction. Hence $v I$ is not contained in any one of $M_{1}, \ldots, M_{n}$.

Corollary 2.5. Let $R$ be an integral domain and I a projective ideal of $R$. If I is contained in only a finite number of maximal ideals of $R$, then $I$ is generated by at most two elements.

Proof. We can assume that $I$ is not the zero ideal. By Lemma 2.4 there exists an element $q \in I^{-1}$ such that $q I$ is not contained in any of the maximal ideals that contain $I$. Since $q I$ is an ideal of $R$ we then have that $I+q I=R$. Hence there exist elements $a, b$ in $I$ such that $1=a+q b$. If $x \in I$, then $x=x a+(q x) b$, and so $I$ is generated by $a$ and $b$.

Corollary 2.6. Let $R$ be an integral domain, $I$ a projective rank one $R$-module and $J$ an ideal of $R$. If $J$ is contained in only a finite number of maximal ideals of $R$, then we have $I / J I \cong R / J$.

Proof. Since $I$ is a finitely generated $R$-submodule of $Q$, there exists a nonzero element $d$ in $R$ such that $d I$ is an ideal of $R$. Then $d I / J d I \cong I / J I$, and so we can assume that $I$ is an ideal of $R$. By Lemma 2.4 there exists an element $q$ in $I^{-1}$ such that $q I$ is not contained in any of the maximal ideals that contain $J$. Thus we have $q I+J=R$, and so $q I \cap J=q I J$. Therefore, we have $R / J=(q I+J) / J \cong q I /(q I \cap J)=q I / q I J \cong I / I J$. 
THEOREM 2.7. Let $R$ be an h-semilocal domain, and I a nonzero projective ideal of $R$. Then,

(1) If $J$ is a nonzero ideal of $R$, we have $I / J I \cong R / J$.

(2) $I$ is generated by two elements, one of which may be chosen arbitrarily.

(3) We have $I^{-1} / R \cong R / I$.

Proof.

(1) This follows from Corollary 2.6.

(2) Let $a$ be a nonzero element of $I$, and let $J=a I^{-1}$. Then $J$ is an ideal of $R$. Hence by Corollary $2.6, I / J I \cong R / J$ is cyclic. Now $J I=a I^{-1} I=R a$. Hence $I$ is generated by $a$ and one other element.

(3) By part (2) $I^{-1}$ is generated by 1 and one other element. Therefore, $I^{-1} / R$ is cyclic. Since $\left(I^{-1}\right)^{-1}=I$, the annihilator of $I^{-1} / R$ is exactly $I$. Thus $I^{-1} / R \cong R / I$.

REMARKs. (1) Theorem 2.7 is of course a classical theorem for Dedekind rings. If $R$ is actually semilocal (i.e., if $R$ has only a finite number of maximal ideals), and if we take $J$ to be the 0 ideal in Corollary 2.6, we obtain the theorem that every projective ideal of $R$ is free on one generator. Thus there is an underlying unity in these two well-known theorems. They are merely special cases of Theorem 2.7 that postulates a weak type of finiteness condition that encompasses both. With the austerity of the assumptions goes a corresponding increased transparency in the nature of the proof.

(2) It is an open question whether Corollary 2.5 is the best result obtainable. In other words, if $I$ is a projective ideal of an arbitrary integral domain, and if $I$ is contained in only a finite number of maximal ideals, can one of the two generators of $I$ be chosen from among any of the nonzero elements of $I$ ? The affirmative answer for $h$-semilocal domains is provided by Theorem 2.7 .

(3) Let $R$ be an $h$-semilocal domain. If $P$ is a projective $R$-module of finite rank $n$, then it follows from [1, Theorem 3] that $P$ is a direct sum of a projective ideal $I$ of $R$ and a free $R$-module of rank $n-1$. By [3, Lemma 1] the only invariants of $P$ are $n$ and the ideal class of $I$. It follows that if $I_{1}, \ldots, I_{n}$ and $J_{1}, \ldots, J_{n}$ are projective ideals of $R$, then $I_{1} \oplus \cdots \oplus I_{n}$ and $J_{1} \oplus \cdots \oplus J_{n}$ are isomorphic if and only if $I_{1} I_{2} \cdots I_{n}$ and $J_{1} J_{2} \cdots J_{n}$ are isomorphic. By the proof of [3, Theorem 2] an $R$ module which is a direct sum of an infinite number of nonzero projective ideals is free.

THEOREM 2.8. Let $R$ be an h-local domain, and $P$ a finitely generated $R$-module. Let $H$ be the completion of $R$. Then the following statements are equivalent:

(1) $P$ is $R$-projective.

(2) $H \otimes_{R} P$ is $H$-free.

Proof. Assume that $P$ is $R$-projective, and let $F$ be a free $R$-module such that rank $F=\operatorname{rank} P$. Let $M$ be a maximal ideal of $R$. Then $P_{M}$ is $R_{M}$-free, and so 
$P_{M} \cong F_{M}$. Hence $K_{M} \otimes_{R} P \cong K_{M} \otimes_{R} P_{M} \cong K_{M} \otimes_{R} F_{M} \cong K_{M} \otimes_{R} F$. Since $R$ is $h$-local, we have $K \cong \sum \oplus K_{M}$, where $M$ ranges over all maximal ideals of $R$. Thus we have $K \otimes_{R} P \cong \sum \oplus\left(K_{M} \otimes_{R} P\right) \cong \sum \oplus\left(K_{M} \otimes_{R} F\right) \cong K \otimes_{R} F$. Therefore, Hom $_{R}\left(K, K \otimes_{R} P\right) \cong$ $\operatorname{Hom}_{R}\left(K, K \otimes_{R} F\right)$. Since $P$ is finitely generated, we have by [8, Proposition 7.2] and [8, Corollary 3.3] that $H \otimes_{R} P \cong \operatorname{Hom}_{R}\left(K, K \otimes_{R} P\right)$. Similarly we have $H \otimes_{R} F \cong \operatorname{Hom}_{R}\left(K, K \otimes_{R} F\right)$. Thus $H \otimes_{R} P \cong H \otimes_{R} F$, and so $H \otimes_{R} P$ is $H$-free.

Conversely, if $H \otimes_{R} P$ is $H$-free, then by [8, Proposition 9.1] we have that $P$ is $R$-projective.

Theorem 2.8 raises the question of which finitely generated projective $\mathrm{H}$ modules are $H$-free? Theorem 3.2 will provide the answer for $h$-local domains. Since Theorem 2.8 will be a special case of Theorem 3.2 , this will give a second proof of Theorem 2.8 .

3. Modules over $h$-local domains. An $R$-module $C$ is said to be cotorsion, if $\operatorname{Hom}_{R}(Q, C)=0$ and $\operatorname{Ext}_{R}^{1}(Q, C)=0$. If $M$ is a maximal ideal of $R$ and $A$ is an $R$-module, we denote $\operatorname{Hom}_{R}\left(R_{M}, A\right)$ by the symbol $A^{M}$. In [8] we showed that $R$ is $h$-local if and only if $T \cong \sum \oplus T_{M}$ for every torsion $R$-module $T$, where $M$ ranges over all maximal ideals of $R$. We then showed that if $R$ is $h$-local and $C$ is a cotorsion $R$-module, then $C=\prod C^{M}$. The next theorem repairs the gap in [8] by showing that these three conditions are all equivalent. It reveals the real reason why $h$-local domains are so useful.

THEOREM 3.1. The following statements are equivalent:

(1) $R$ is an h-local domain.

(2) $T \cong \sum \oplus T_{M}$ for all torsion $R$-modules $T$, where $M$ ranges over all maximal ideals of $R$.

(3) $C \cong \prod C^{M}$ for all cotorsion $R$-modules $C$, where $M$ ranges over all maximal ideals of $R$.

Proof. We have already proved ([8, Theorem 8.5$]$ and [8, Corollary 8.6]) that $(1) \Rightarrow(2)$ and $(2) \Rightarrow(3)$. It remains to prove that $(3) \Rightarrow(1)$. Hence assume that (3) holds.

Let $P$ be a nonzero prime ideal of $R$. We will show first that $P$ is contained in only one maximal ideal of $R$. Now $R / P$ is torsion of bounded order, and hence is cotorsion. Thus by assumption we have $R / P \cong \Pi(R / P)^{M}$. If there are at least two nonzero components in this product, then there exist ideals $A$ and $B$ of $R$, each properly containing $P$, such that $R / P=A / P \oplus B / P$. Thus we have $A \cap B=P$. Since $P$ is a prime ideal, this is a contradiction. Hence there exists a maximal ideal $M$ of $R$ such that $R / P \cong(R / P)^{M}$, and $(R / P)^{N}=0$ for every other maximal ideal $N$ of $R$. Thus we have $R_{M} / P_{M} \cong(R / P)_{M} \cong\left[(R / P)^{M}\right]_{M} \cong(R / P)^{M} \cong R / P$. Hence $P_{M} \neq R_{M}$, and so $P \subset M$. 
Let $N$ be a maximal ideal of $R$ different from $M$. We must show that $P$ is not contained in $N$. Now $R_{N} / P_{N}$ is torsion of bounded order as an $R$-module, and so is a cotorsion $R$-module. Thus we have $R_{N} / P_{N} \cong \prod\left(R_{N} / P_{N}\right)^{L}$, where $L$ ranges over all maximal ideals of $R$. But $\left(R_{N} / P_{N}\right)^{L}$ is an $R_{N}$-module; and $R_{N} / P_{N}$ is a local $R_{N^{-}}$ module, and hence indecomposable. Therefore, $\left(R_{N} / P_{N}\right)^{L}=0$ for every $L \neq N$. In particular, $\left(R_{N} / P_{N}\right)^{M}=0$.

Suppose that we have $P \subset N$. We define a map $\varphi:\left[(R / P)^{M}\right]_{N} \rightarrow\left(R_{N} / P_{N}\right)^{M}$ as follows. We have $\left[(R / P)^{M}\right]_{N}=\operatorname{Hom}_{R}\left(R_{M}, R / P\right)_{N}$, and

$$
\left(R_{N} / P_{N}\right)^{M} \cong \operatorname{Hom}_{R_{N}}\left(R_{M} \otimes_{R} R_{N}, R_{N} / P_{N}\right)
$$

Let $f \in \operatorname{Hom}_{R}\left(R_{M}, R / P\right), x \in R_{M}$, $s$ and $t \in R-N$; then set

$$
[\varphi(f \otimes(1 / s))](x \otimes(1 / t))=f(x) \otimes(1 / s t)
$$

Suppose that $f \otimes(1 / s) \in \operatorname{Ker} \varphi$; then for each $x \in R_{M}$ there exists $u_{x} \in R-N$ such that $u_{x} f(x)=0$. But $f(x) \in R / P$, and $u_{x} \notin P$. Therefore, $f(x)=0$ for all $x \in R_{M}$, and so $f=0$. Thus we have $\operatorname{Ker} \varphi=0$. But we have seen above that $\left(R_{N} / P_{N}\right)^{M}=0$. Hence we have $\left[(R / P)^{M}\right]_{N}=0$, also. Therefore, $R_{N} / P_{N} \cong(R / P)_{N} \cong\left[(R / P)^{M}\right]_{N}=0$. This contradicts $P \subset N$, and so we have that $M$ is the only maximal ideal of $R$ containing $P$.

Let $M$ and $N$ be two distinct maximal ideals of $R$. Then by what we have just shown, $M \cap N$ contains no nonzero prime ideal of $R$. Hence by [8, Corollary 8.2] we have $R_{M} \otimes_{R} R_{N}=Q$.

Let $I$ be a nonzero ideal of $R$, and let $T=R / I$. We must show that $I$ is contained in only a finite number of maximal ideals of $R$. Let $\mathscr{M}$ be the collection of maximal ideals $M$ of $R$ such that $T^{M} \neq 0$. We will show first that $\mathscr{M}$ is a finite set. Since $T$ is a cotorsion $R$-module, we have $T \cong \prod T^{M}, M \in \mathscr{M}$. Let $S=\sum \oplus T^{M}, M \in \mathscr{M}$. Then $S \subset T$, and so $S$ is torsion of bounded order. Therefore, $S$ is a cotorsion $R$ module, and we have $S \cong \prod S^{N}$, where $N$ ranges over all maximal ideals of $R$. If $N$ is a maximal ideal such that $N \notin \mathscr{M}$, then $S^{N} \subset T^{N}=0$. Therefore, we have $S \cong \prod S^{M}, M \in \mathscr{M}$.

Let $N$ and $M$ be two distinct maximal ideals of $R$. Then

$$
\left(T^{M}\right)^{N} \cong \operatorname{Hom}_{R}\left(R_{N} \otimes_{R} R_{M}, T\right) \cong \operatorname{Hom}_{R}(Q, T)=0
$$

Thus if $N \in \mathscr{M}$, we have $S^{N}=\operatorname{Hom}_{R}\left(R_{N}, \sum_{M \in \mathscr{M}} \oplus T^{M}\right) \cong\left(T^{N}\right)^{N} \cong T^{N}$. Therefore, $S \cong \prod_{M \in \mathscr{M}} S^{M} \cong \prod_{M \in \mathscr{M}} T^{M} \cong T$. We also have $\left(T^{M}\right)_{N} \cong\left(\left[T^{M}\right]_{M}\right)_{N} \cong T^{M} \otimes_{R} Q=0$. Therefore, if $N \notin \mathscr{M}$, we have $S_{N} \cong \sum_{M \in \mathscr{M}} \oplus\left(T^{M}\right)_{N}=0$. Since $S \cong T$, we also have $T_{N}=0$, and hence $(T / S)_{N}=0$. Now let $M \in \mathscr{M}$. Then $T=T^{M} \oplus A, S=T^{M} \oplus B$, where $B \subset A$ are submodules of $T$. Hence $T_{M} \cong\left(T^{M}\right)_{M} \oplus A_{M} \cong T^{M} \oplus A_{M}$. But $T_{M} \cong R_{M} / I_{M}$ is a local $R_{M}$-module and hence is indecomposable. Therefore, we have $A_{M}=0$, and it 
follows that $(A / B)_{M}=0$ also. Now $T / S \cong A / B$, and so $(T / S)_{M}=0$. We have thus shown that $(T / S)_{N}=0$ for every maximal ideal $N$ of $R$. Hence we have $S=T$. But this means that the set $\mathscr{M}$ is finite.

If $N \notin \mathscr{M}$, then we have seen above that $R_{N} / I_{N}=T_{N}=0$. This says that $I$ is not contained in $N$, and therefore, $I$ is contained in only the maximal ideals belonging to $\mathscr{M}$, which is a finite set. Hence $I$ is contained in only a finite number of maximal ideals of $R$. Thus $R$ is an $h$-local ring, and the proof of the theorem is complete.

Definition. Let $R$ be an $h$-local ring, $H$ its completion, and $M$ a maximal ideal of $R$. Then $H M$ is a maximal ideal of $H$ and $H / H M \cong R / M$ (see [8, Proposition 5.10]). Let $P$ be a projective $H$-module, and define $\operatorname{rank}_{M} P$ to be the dimension of $P / M P$ over $R / M$ (or over $H / H M$ ). If $P$ is a finitely generated $H$-module, then $\operatorname{rank}_{M} P$ is finite.

THEOREM 3.2. Let $R$ be an h-local domain, and $H$ its completion. Let $P$ be a finitely generated, projective $\mathrm{H}$-module. Then $\mathrm{P}$ is isomorphic to a finite direct sum of principal ideals of H.P is a free $H$-module if and only if $\operatorname{rank}_{M} P$ is constant for all maximal ideals $M$ of $R$.

Proof. Since $P$ is a direct summand of a finitely generated free $H$-module, $P$ is a cotorsion $R$-module. Thus by Theorem 3.1 we have $P \cong \prod P^{M}$, where $M$ ranges over all maximal ideals of $R$. By [8, Theorem 8.5] we have $H=\prod H(M)$, where $M$ ranges over all maximal ideals of $R$ and $H(M)=\operatorname{Hom}_{R_{M}}\left(K_{M}, K_{M}\right)$. Since $H(M) / R_{M}$ is torsion-free and divisible, we have $P^{M} \cong \operatorname{Hom}_{R}(H(M), P)$, and so $P^{M}$ is an $H(M)$-module. If $N$ and $M$ are different maximal ideals of $R$, we have by [8, Lemma 8.3] that $\operatorname{Hom}_{R}\left(H(M), P^{N}\right)=0$. This shows that $H=\Pi H(M)$ acts componentwise on $P=\prod P^{M}$, and that $P^{M}$ is the $H(M)$-component of $P$. Therefore, $P^{M}$ is a finitely generated, projective $H(M)$-module.

Since $P$ is a direct summand of a finitely generated, free $H$-module, $\operatorname{rank}_{M} P$ is bounded. Let $n=\max \left(\operatorname{rank}_{M} P\right)$, where $M$ ranges over all maximal ideals of $R$. Let $A=\prod_{N \neq M} H(N)$, so that $H=H(M) \oplus A$. Then $R / M \cong H / M H \cong H(M) / M H(M)$ $\oplus A / M A$, and so we have $A=M A$. Hence if $B=\prod_{N \neq M} P^{N}$, we have $M B=B$. Thus $P / M P \cong P^{M} / M P^{M}$, and so $\operatorname{rank}_{M} P^{M}=\operatorname{rank}_{M} P \leqq n$.

It will follow from Proposition 4.1 of the next section, that since $R_{M}$ is a local ring, $H(M)$ is a local ring also. Thus $P^{M}$, being a projective $H(M)$-module, is a free $H(M)$-module of rank $\leqq n$. Therefore, $P^{M}=\sum_{i=1}^{n} \oplus A_{i, M}$, where $A_{i, M}=0$ or $A_{i, M} \cong H(M)$. Thus we have $P \cong \prod P^{M} \cong \prod_{M}\left(\sum_{i=1}^{n} \oplus A_{i, M}\right) \cong \sum_{i=1}^{n} \oplus\left(\prod_{M} A_{i, M}\right)$. Now $\prod_{M} A_{i, M}$ is isomorphic to $H$ if $A_{i, M} \neq 0$ for every $M$, and it is isomorphic to a direct summand of $H$, otherwise. Hence $P$ is isomorphic to a finite direct sum of principal ideals of $H$. It is also clear that $P$ is a free $H$-module if and only if $\operatorname{rank}_{M} P=n$ for every maximal ideal $M$ of $R$. 
TheOREM 3.3. Let $R$ be an h-local domain, and $C$ an $R$-module. Then inj. $\operatorname{dim}_{\cdot_{R}} C=$ $\sup _{M}$ inj. $\operatorname{dim}_{R_{M}} C_{M}$, where $M$ ranges over all maximal ideals of $R$. Thus gl. dim. $R=$ $\sup _{M}$ gl. dim. $R_{M}$.

Proof. We will prove first that inj. $\operatorname{dim}_{\cdot_{R}} C \leqq \sup _{M} \operatorname{inj.} \operatorname{dim}_{\cdot_{R_{M}}} C_{M}$. Hence we can assume that $\sup _{M}$ inj. $\operatorname{dim}_{R_{M}} C_{M}=n<\infty$.

Case I. $n=0$. In this case $C_{M}$ is an injective $R_{M}$-module for all maximal ideals $M$ of $R$. Hence by [2, Chapter VI, Proposition 4.1.3], $C_{M}$ is an injective $R$-module for all $M$. Let $T$ be the torsion submodule of $C$, and let $S=C / T$. Then $T_{M}$ is the torsion submodule of $C_{M}$, and so $C_{M} \cong T_{M} \oplus S_{M}$. Hence $S_{M}$ is torsion-free and divisible. Since $S \subset S_{M} \subset Q \otimes_{R} S$, it follows that $S_{M}=Q \otimes_{R} S$. Thus $S=\bigcap S_{M}=Q \otimes_{R} S$, and so $S$ is an injective $R$-module. It suffices then in this case to prove that $T$ is an injective $R$-module, for then $C \cong T \oplus S$ will be an injective $R$-module.

Let $I$ be a nonzero ideal of $R$ and $N$ a maximal ideal of $R$. Consider an exact sequence of the form:

$$
0 \rightarrow T \rightarrow A \rightarrow R_{N} / I_{N} \rightarrow 0 .
$$

Then $A$ is a torsion $R$-module, and we have $A=\sum \oplus A_{M}$ and $T=\sum \oplus T_{M}$. From exact sequence (*) we see that we have $A_{M} / T_{M} \cong\left(R_{N} / I_{N}\right)_{M}$. If $M \neq N$, we have $\left(R_{N} / I_{N}\right)_{M} \cong$ $R / I \otimes_{R} Q=0$ by [8, Corollary 8.2$]$. Thus $T_{M}=A_{M}$ for all $M \neq N$. Hence exact sequence $(*)$ splits if and only if the following exact sequence splits:

$$
0 \rightarrow T_{N} \rightarrow A_{N} \rightarrow R_{N} / I_{N} \rightarrow 0 .
$$

But this sequence splits, because $T_{N}$ is an injective $R_{N}$-module. Therefore $\left({ }^{*}\right)$ splits, and so we have $\operatorname{Ext}_{R}^{1}\left(R_{N} / I_{N}, T\right)=0$. By Theorem 3.1 we have $R / I \cong \sum_{N} \oplus R_{N} / I_{N}$, and so we have $\operatorname{Ext}_{R}^{1}(R / I, T) \cong \prod_{N} \operatorname{Ext}_{R}^{1}\left(R_{N} / I_{N}, T\right)=0$. Therefore, $T$ is an injective $R$-module.

Case II: $n>0$. Take an exact sequence:

$$
0 \rightarrow C \rightarrow E_{0} \rightarrow E_{1} \rightarrow \cdots \rightarrow \dot{E_{n-1}} \rightarrow D \rightarrow 0,
$$

where each $E_{i}$ is an injective $R$-module. Then for each maximal ideal $M$ of $R$ we have an exact sequence:

$$
0 \rightarrow C_{M} \rightarrow\left(E_{0}\right)_{M} \rightarrow\left(E_{1}\right)_{M} \rightarrow \cdots \rightarrow\left(E_{n-1}\right)_{M} \rightarrow D_{M} \rightarrow 0 .
$$

Now $E_{i}$ is a direct sum of a torsion injective $R$-module and a torsion-free injective $R$-module. Hence using Theorem 3.1 we see that $\left(E_{i}\right)_{M}$ is a direct summand of $E_{i}$, and so is $R$-injective. Thus by [2, Chapter VI, Proposition 4.1.3], $\left(E_{i}\right)_{M}$ is an injective $R_{M}$-module. Since inj. $\operatorname{dim}_{R_{M}} C_{M} \leqq n$, it follows that $D_{M}$ is an injective $R_{M^{-}}$ module for all $M$. Therefore, by Case I, $D$ is an injective $R$-module. Thus we see from exact sequence $\left({ }^{* *}\right)$ that inj. $\operatorname{dim}_{{ }_{R}} C \leqq n$. 
We next prove that $\sup _{M} \operatorname{inj} \cdot \operatorname{dim}_{R_{M}} C_{M} \leqq \operatorname{inj} . \operatorname{dim}_{\cdot_{R}} C$; and for this we may assume that inj. $\operatorname{dim}_{{ }_{R}} C=n<\infty$. Then we have an exact sequence of the form (**) with $D=E_{n}$, and $E_{i}$ an injective $R$-module for all $i \geqq 0$. As above it follows that $\left(E_{i}\right)_{M}$ is an injective $R_{M}$-module for all $i \geqq 0$. We then have an exact sequence of the form $(* * *)$ for each maximal ideal $M$, with $D_{M}=\left(E_{n}\right)_{M}$. Therefore,

$$
\underset{M}{\sup } \underset{R_{M}}{\operatorname{inj}} \operatorname{dim} . C_{M} \leqq n,
$$

and the theorem is proved.

\section{Local domains.}

Proposition 4.1. Let $R$ be a local domain with maximal ideal $M$, and let $H$ be the completion of $R$. Then $H$ is a commutative local ring with maximal ideal $H M$, and $H / H M \cong R / M$.

Proof. By [8, Proposition 5.10], $H M$ is a maximal ideal of $R$ and $H / H M \cong R / M$. $H$ is a commutative ring by [8, Proposition 5.1]. Suppose that $J$ is an ideal of $H$ that is not contained in $H M$. Then $H=H M+J$, and so there exist $g \in H M$ and $p \in J$ such that $1=g+p$. Now we have $g=\sum_{i=1}^{n} h_{i} m_{i}$, where $h_{i} \in H$ and $m_{i} \in M$ for $i=1, \ldots, n$. Let $s$ be a nonzero element of $R$, and set $y=[(1 / s)+R] \in K$. Then $h_{i}(y)$ is an element of $K$ that is annihilated by $s$, and so $h_{i}(y) \in R y$. It follows that if $x$ is any element of $K$, then there exists an element $r_{i, x} \in R$ such that $h_{i}(x)=r_{i, x} x$. Hence $g(x)=\sum_{i=1}^{n} h_{i}\left(m_{i} x\right)=\sum_{i=1}^{n} r_{i, x} m_{i} x=m_{x} x$, where $m_{x} \in M$. Therefore,

$$
p(x)=x-g(x)=x-m_{x} x=\left(1-m_{x}\right) x .
$$

Let $u_{x}=1-m_{x}$; then $u_{x}$ is a. unit in $R$ and we have $p(x)=u_{x} x$. It follows that $p$ is an automorphism of $K$, and so $p^{-1} \in H$. Thus $J=H$, which shows that $H$ is a local ring with maximal ideal $H M$.

Corollary 4.2. Let $R$ be a local domain. Then $K$ is an indecomposable $R$-module.

Proof. Since $H$ is a local ring by Proposition 4.1, it can have no idempotents other than the identity. Therefore $K$ is indecomposable.

REMARKS. It is not true in general that if $R$ is a local domain and $I$ is an ideal of $R$, then $K \otimes_{R} I$ is indecomposable, as we shall see in Theorem 7.4.

COROllary 4.3. Let $R$ be an h-local domain, and $H$ its completion. Then $H$ is a direct product of commutative, local rings. 
Proof. By [8, Theorem 8.5] we have $H \cong \prod H(M)$, where

$$
H(M)=\operatorname{Hom}_{R_{M}}\left(K_{M}, K_{M}\right)
$$

for all maximal ideals $M$ of $R$. By Proposition 4.1, $H(M)$ is a commutative local ring.

LEMmA 4.4. Let $R$ be an integral domain and $H$ its completion. Let $J$ be an ideal of $R$ such that $J \cap R=0$. Let $P$ be an ideal of $H$ that is maximal with respect to the properties that $J \subset P$ and $P \cap R=0$. Then $P$ is a prime ideal of $H$; and if $P$ is not a cotorsion $R$-module, then $P$ is a maximal ideal of $R$ such that $H / P$ is torsion-free and divisible over $R$.

Proof. It is clear from an application of Zorn's Lemma that there exist ideals $P$ of $H$ that are maximal with respect to the properties that $J \subset P$ and $P \cap R=0$. Let $P$ be such an ideal. Let $a, b$ be elements of $H$ such that $a b \in P$. Suppose that $a \notin P$ and $b \notin P$. Then we have $r=p+h a$ and $s=q+k b$, where $r, s$ are nonzero elements of $R, p, q \in P$, and $h, k \in H$. Then $r s \in P \cap R=0$, and so $r=0$ or $s=0$, which is a contradiction. Thus either $a \in P$ or $b \in P$; and so $P$ is a prime ideal of $H$.

Suppose that $P$ is not a cotorsion $R$-module. Assume that $P$ is not a maximal ideal of $H$. Then there is an ideal $A$ of $H$ that is properly between $P$ and $H$. But then $A \cap R \neq 0$, and so by [8, Proposition 7.8], $A$ is a cotorsion $R$-module. Let $S$ be the intersection of all cotorsion submodules of $H$ that contain $P$. Now $H / P$ is a torsion-free $R$-module, since $P$ is a prime ideal of $H$ and $P \cap R=0$. Therefore [8, Proposition 2.8] applies, and we have that $S / P$ is torsion-free and divisible, and that $S$ is a cotorsion submodule of $H$. Thus $S$ is an ideal of $H$, and $S \subset A$. Therefore, $S \neq P$ and $S \neq H$. Hence $S \cap R \neq 0$, and so there exists a nonzero element $r \in S \cap R$. By [8, Proposition 7.8], $P+H r$ is a cotorsion $R$-module, and so $S \subset P+H r$. But of course $P+H r \subset S$, and so we have $S=P+H r$. Therefore,

$$
S / P=(P+H r) / P \cong H r /(P \cap H r)=H r / P r \cong H / P .
$$

Thus $H / P$ is torsion-free and divisible. But by [8, Proposition 2.8] this implies that $H=S$. This contradiction shows that $P$ is a maximal ideal of $H$. It then follows from [8, Proposition 7.8] that $H / P$ is torsion-free and divisible.

TheOREM 4.5. Let $R$ be a local domain. Then the following statements are equivalent:

(1) Every proper $R$-submodule of $K$ is $h$-reduced.

(2) If $J$ is a nonzero ideal of $H$, then $J \cap R \neq 0$.

(3) If $J$ is any ideal of $H$ and $I=J \cap R$, then $J=H I$.

(4) Every nonzero element of $H$ is an epimorphism of $K$.

If any of these conditions hold, then $H$ is an integral domain. 
Proof. (1) $\Rightarrow(2)$. Suppose that $J$ is a nonzero ideal of $H$, and that $J \cap R=0$. By Proposition 4.1, $H$ is a local ring with maximal ideal $H M$, where $M$ is the maximal ideal of $R$, and $H / H M \cong R / M$. Let $P$ be an ideal of $H$ maximal with respect to the properties that $J \subset P$ and $P \cap R=0$. Since $P \neq H M$, we have by Lemma 4.4 that $P$ is a cotorsion $R$-module and $H / P$ is torsion-free. Thus we have an exact sequence:

$$
0 \longrightarrow K \otimes_{R} P \stackrel{f}{\longrightarrow} K \otimes_{R} H \longrightarrow K \otimes_{R} H / P \longrightarrow 0 .
$$

Since $K \otimes_{R} H \cong K$ by [8, Proposition 5.5], we have by assumption that either $f$ is an epimorphism, or $f=0$. If $f=0$, then $K \otimes_{R} P=0$; and so $P$ is torsion-free and divisible, which contradicts the fact that $P$ is cotorsion. Hence $f$ is an epimorphism. But this implies that $K \otimes_{R} H / P=0$, and so $H / P$ is torsion-free and divisible. But this also contradicts the fact that $P$ is cotorsion. Thus we must have that $J \cap R \neq 0$.

$(2) \Leftrightarrow(3)$. This equivalence has been proved in [8, Proposition 7.8].

$(2) \Rightarrow(4)$. Let $g$ be a nonzero element of $H$. Then by assumption there exists a nonzero element $r$ in $H g \cap R$. Thus $r=k g$, where $k \in H$. Now $K=r K=g(k(K)) \subset$ $g(K) \subset K$. Hence $K=g(K)$, and $g$ is an epimorphism.

(4) $\Rightarrow(1)$. Let $L$ be a nonzero, $h$-divisible submodule of $K$. Then by [8, Lemma 3.1] we have that $L \cong K \bigotimes_{R} \operatorname{Hom}_{R}(K, L)$. Therefore, $\operatorname{Hom}_{R}(K, L) \neq 0$. Now $\operatorname{Hom}_{R}(K, L) \subset H$, and every element of $H$ is an epimorphism on $K$. Thus $L=K$, and so $K$ has no proper, $h$-divisible submodules.

Suppose that (4) holds and that $h, k$ are elements of $H$ such that $h k=0$. Since $h$ and $k$ are epimorphisms or zero, we must have that either $h=0$ or $k=0$. Thus $H$ is an integral domain.

Definition. We will say that a local domain is closed, if it satisfies the conditions of Theorem 4.5.

Examples of closed, local domains.

(1) A valuation ring is closed, since the submodules of $K$ are linearly ordered.

(2) Let $R$ be an analytically irreducible, Noetherian, local domain of Krull dimension one. Then the completion of $R$ in the maximal ideal topology is equal to $H$ (see [8, p. 49]). By definition, $H$ is then an integral domain. Since the Krull dimension of $H$ is equal to the Krull dimension of $R$, which is one, the only prime ideals of $H$ are 0 and $H M$ (where $M$ is the maximal ideal of $R$ ). Hence by Lemma 4.4 we have $J \cap R \neq 0$ for every nonzero ideal $J$ of $H$. Thus $R$ is a closed, local domain.

THEOREM 4.6. Let $R$ be a closed, local domain. Then we have the following:

(1) If $\widetilde{Q}$ is the quotient field of $H$, then $\tilde{Q} \cong H \otimes_{R} Q$ and $\tilde{Q} / H \cong K$.

(2) If $I$ is a nonzero ideal of $R$, then $\widetilde{Q} / H I \cong Q / I$ and $H / H I \cong R / I$.

(3) $H$ is complete in the H-topology.

(4) The categories of torsion R-modules and torsion H-modules are identical. 
(5) The categories of cotorsion R-modules and cotorsion $H$-modules are identical.

(6) If $S$ is any $H$-module, then inj. $\operatorname{dim}_{\cdot_{R}} S=\operatorname{inj.} \operatorname{dim}_{\cdot_{H}} S$, and w. $\operatorname{dim}_{{ }_{R}} S=$ w. $\operatorname{dim}_{\cdot_{H}} S$.

(7) gl. dim. $R=$ gl. dim. $H$ and w. gl. dim. $R=$ w. gl. dim. $H$.

Proof. Let $J$ be a nonzero ideal of $H$ and $I=J \cap R$. By Theorem 4.5 we have $J=H I$; and by [8, Proposition 5.10] we have $H / J \cong R / I$. By [8, Proposition 5.5] we have $\operatorname{Ext}_{H}^{n}\left(H / J, H \otimes_{R} Q\right) \cong \operatorname{Ext}_{R}^{n}\left(R / I, H \otimes_{R} Q\right)=0$ for every $n \geqq 0$. Thus $H \otimes_{R} Q$ is an injective $H$-module. Consider the exact sequence:

$$
0 \rightarrow R \rightarrow Q \rightarrow K \rightarrow 0
$$

If we tensor this sequence with $H$ we obtain the exact sequence:

$$
0 \rightarrow H \rightarrow H \otimes_{R} Q \rightarrow K \rightarrow 0
$$

by [8, Proposition 5.5]. Since $K$ is torsion as an $R$-module, it is a fortiori torsion as an $H$-module. Thus $H \otimes_{R} Q$ is the injective envelope of $H$, and so $H \otimes_{R} Q \cong \widetilde{Q}$, the quotient field of $H$. From this last exact sequence we clearly have $\widetilde{Q} / H \cong K$.

Now $J \cong H \otimes_{R} I$ by [8, Proposition 5.10] and [8, Proposition 7.8]. Thus we have $\widetilde{Q} / H I \cong K \otimes_{H} H I \cong K \otimes_{H}\left(H \otimes_{R} I\right) \cong K \otimes_{R} I \cong Q / I$.

It is clear from Theorem 4.5 that the $R$-topology and the $H$-topology on an $H$-module are the same. In particular, $H$ is complete in the $H$-topology.

It follows from [8, Proposition 5.5] that the categories of torsion $R$-modules and torsion $H$-modules are the same. And it follows from [8, Proposition 5.7] that the categories of cotorsion $R$-modules and cotorsion $H$-modules are the same.

If $S$ is any $H$-module, then the injective dimension of $S$ over $R$ or over $H$ is determined by the vanishing of $\operatorname{Ext}_{R}^{n}(T, S)$ or $\operatorname{Ext}_{H}^{n}(T, S)$, respectively, where $T$ ranges over all torsion $R$-modules (which are the same as all torsion $H$-modules). These two Ext modules are isomorphic by [8, Proposition 5.5]. Thus we have inj. $\operatorname{dim}_{\cdot_{R}} S=\mathrm{inj} . \operatorname{dim}_{\cdot_{H}} S$. A similar argument shows that w. $\operatorname{dim}_{\cdot_{R}} S=$ w. $\operatorname{dim}_{\cdot_{R}} S$.

The weak global dimension of an integral domain is completely determined by its torsion modules. Thus by [2, Chapter VI, Proposition 4.1.2] we have w. gl. $\operatorname{dim} . R=$ w. gl. dim. $H$. By [8, Proposition 3.5] we have that gl. $\operatorname{dim} . R=$ sup inj. $\operatorname{dim}_{._{R}} A$, where $A$ ranges over all torsion-free, cotorsion $R$-modules $A$. A similar statement is true for $H$. Since these two categories are identical, and since the injective dimensions of the modules in this category are the same whether considered over $R$ or over $H$, we have that $\mathrm{gl}$. dim. $R=\mathrm{gl}$. dim. $H$.

Definition. Almost maximal valuation rings and maximal valuation rings have been defined in terms of the existence of solutions of systems of simultaneous congruences. However, in view of [6, Theorem 4] and [6, Theorem 9] we may give the following alternative definitions. A valuation ring $R$ is called almost maximal if $K$ 
is injective; it is called maximal if it is both almost maximal and complete in the $R$-topology.

Proposition 4.7. Let $R$ be an integral domain and $H$ its completion. Then $R$ is an almost maximal valuation ring if and only if $H$ is a maximal valuation ring.

Proof. Suppose that $H$ is a valuation ring. Then by [8, Proposition 5.10] the ideals of $R$ are linearly ordered. Hence $R$ is a valuation ring. Thus we may assume that $R$ is a valuation ring. Then by [8, Proposition 8.8], $H$ is a valuation ring. If $\tilde{Q}$ is the quotient field of $H$, then by Theorem $4.6, \tilde{Q} / H \cong K$ and inj. $\operatorname{dim}_{\cdot_{R}} K=$ inj. $\operatorname{dim}_{{ }_{H}} K$. The proposition now follows from the definitions.

Proposition 4.8. Let $R$ be a valuation ring and $H$ its completion. Then $R$ is almost maximal if and only if there exists an injective $R$-module $C$ such that $H \cong \operatorname{Hom}_{R}(C, C)$.

Proof. If $R$ is almost maximal, then $K$ is injective and $H \cong \operatorname{Hom}_{R}(K, K)$. Conversely, suppose that there exists an injective $R$-module $C$ such that $H \cong \operatorname{Hom}_{R}(C, C)$. Since $H$ is $h$-reduced, $C$ is a torsion $R$-module. Thus by Theorem 4.6, $C$ is an injective $H$-module and $H \cong \operatorname{Hom}_{H}(C, C)$. Let $A$ be any $H$-module. Since $H$ is a valuation ring, we have w. gl. $\operatorname{dim} . H=1$, and so $\operatorname{Tor}_{2}^{H}(A, C)=0$. Thus using [2, Chapter VI, Proposition 5.1] we have $\operatorname{Ext}_{H}^{2}(A, H) \cong \operatorname{Ext}_{H}^{2}\left(A, \operatorname{Hom}_{H}(C, C)\right) \cong$ $\operatorname{Hom}_{H}\left(\operatorname{Tor}_{2}^{H}(A, C), C\right)=0$. Therefore, inj. $\operatorname{dim}_{\cdot_{H}} H=1$, and so $K$ is $H$-injective. Hence by Theorem 4.6, $K$ is $R$-injective, and $R$ is an almost maximal valuation ring.

5. Decomposable torsion modules. The fundamental theorem of Abelian groups shows that every finitely generated torsion module over a Dedekind ring is a direct sum of cyclic modules. I. Kaplansky has extended this result to include almost maximal valuation rings ([3, Theorem 14]). The present author has proved that if a Noetherian integral domain has this property, then it must be a Dedekind ring ([6, Proposition 15]). The main purpose of this section is to generalize the above result of Kaplansky and prove its converse (see Theorem 5.7). The story does not end here, for it would be desirable to remove the assumption in Theorem 5.7 that $R$ is an $h$-local domain.

Proposition 5.1. Let $R$ be an h-local Prufer domain, and let $A$ be a direct sum of cyclic R-modules. Then any torsion and finitely generated $R$-submodule of $A$ is again a direct sum of cyclic $R$-modules.

Proof. Since $R$ is a Prufer domain, the torsion submodule of $A$ is a direct summand of $A$. Thus we may assume that $A$ is a direct sum of a finite number of cyclic torsion $R$-modules. By Theorem 3.1 there are a finite number of maximal ideals 
$M_{1}, \ldots, M_{n}$ of $R$ such that $A \cong A_{M_{1}} \oplus \cdots \oplus A_{M_{n}}$. Each $A_{M_{i}}$ is a finite direct sum of cyclic torsion $R_{M_{i}}$-modules. Let $B$ be an $R$-submodule of $A$. Then $B=B_{M_{1}} \oplus \cdots$ $\oplus B_{M_{n}}$, and $B_{M_{i}} \subset A_{M_{i}}$. By Lemma 2.1 the $R$-structure and the $R_{M_{i}}$-structure of $A_{M_{i}}$ and $B_{M_{i}}$ are the same. Thus we may assume without loss of generality that $R$ is a local domain. But then $R$ is a valuation ring, and the proposition is an immediate consequence of $[6$, Proposition 14].

Proposition 5.2. Let $R$ be an h-local Prufer domain, and let $B$ be a finitely generated $R$-module. Let $E(B)$ be the injective envelope of $B$. Then $E(B)$ is a finite direct sum of indecomposable injective $R$-modules.

Proof. Since $R$ is a Prufer domain, the torsion submodule of $B$ is a direct summand of $B$. Hence $E(B)$ is the direct sum of the injective envelope of the torsion submodule of $B$ and of a finite number of copies of $Q$. Thus we may assume that $B$ is a torsion $R$-module. By Theorem 3.1 there are a finite number of maximal ideals $M_{1}, \ldots, M_{n}$ of $R$ such that $B \cong B_{M_{1}} \oplus \cdots \oplus B_{M_{n}}$. If $M$ is a maximal ideal of $R$ and $C$ an injective $R_{M}$-module, then by [2, Chapter VI, Proposition 4.1.3] $C$ is also an injective $R$-module. Hence we may assume that $R$ is a local ring. But then $R$ is a valuation ring, and so the proposition is an immediate consequence of [6, Proposition 1].

Definition. Let $R$ be an integral domain and $A$ a nonempty subset of an $R$ module. Then we will define $O(A)$ to be the annihilator of $A$; i.e., $O(A)=$ $\{r \in R \mid r A=0\}$.

LEMMA 5.3. Let $R$ be a valuation ring and $B$ an $R$-module. Let $y$ be a nonzero element of $B$ and $r$ an element of $R$ such that $r y \neq 0$. Then $r O(r y)=O(y)$.

Proof. Since $r \notin O(y)$, we have $O(y) \subset R r$. Thus $O(y)=J r$, where $J$ is an ideal of $R$. Let $a \in O(r y)$; then $0=a r y$, and so $a r \in O(y)$. Therefore, $a \in J$ and we have $O(r y) \subset J$. Conversely, let $a \in J$. Then $a r \in O(y)$, and so $a \in O(r y)$. Thus $J \subset O(r y)$, and we have $J=O(r y)$. Hence $O(y)=J r=r O(r y)$.

LEMMA 5.4. Let $R$ be a valuation ring, and let $E(K)$ be the injective envelope of $K$. Then $E(K)$ is an indecomposable injective $R$-module. Furthermore, if $y \in E(K)$, then $O(y)$ is a principal ideal of $R$.

Proof. Let $S$ and $T$ be nonzero submodules of $E(K)$. Then $S \cap K \neq 0$ and $T \cap K \neq 0$, since $E(K)$ is an essential extension of $K$. Now the submodules of $K$ are linearly ordered, and so we have that $(S \cap K) \cap(T \cap K) \neq 0$. Therefore, $S \cap T \neq 0$, and so $E(K)$ is an indecomposable, injective $R$-module.

Let $y$ be a nonzero element of $E(K)$. Take a nonzero element $a$ in $R$ which is not a unit in $R$, and let $v=(1 / a)+R$, a nonzero element of $K$. Then $R y \cap R v \neq 0$, and 
so there exist elements $r, s$ in $R$ such that $r y=s v \neq 0$. Thus by Lemma 5.3 we have $O(y)=r O(r y)=r O(s v)=(r / s) O(v)$. Now $O(v)=R a$, and so $O(y)=R(r a / s)$ is a principal ideal of $R$.

The following lemma has been proved by I. Kaplansky in [3, Theorem 14$]\left({ }^{1}\right)$. We give an independent proof that is based on the properties of indecomposable, injective modules.

LEMMA 5.5. Let $R$ be a valuation ring. Then $R$ is an almost maximal valuation ring if and only if every finitely generated $R$-module is a direct sum of cyclic $R$-modules.

Proof. Assume that $R$ is an almost maximal valuation ring, and let $B$ be a finitely generated $R$-module. By Proposition 5.2 the injective envelope $E(B)$ is a finite direct sum of indecomposable, injective $R$-modules. By [6, Theorem 4] every indecomposable, injective $R$-module is of the form $Q / I$, where $I$ is an ideal of $R$. Now $Q / I$ has the property that every finitely generated $R$-submodule is cyclic. Thus $B$ is a finitely generated $R$-submodule of a direct sum of cyclic $R$-modules. Since $B$ is a direct sum of its torsion submodule and a free $R$-module, we can assume without loss of generality that $B$ is a torsion $R$-module. It then follows from Proposition 5.1 that $B$ itself is a direct sum of cyclic $R$-modules.

Conversely assume that every finitely generated $R$-module is a direct sum of cyclic $R$-modules. By our definitions, to prove that $R$ is an almost maximal valuation ring it is sufficient to prove that $E(K)=K$, where $E(K)$ is the injective envelope of $K$. Suppose that $E(K) \neq K$, and take $y \in E(K)-K$. Then by Lemma 5.4, $O(y)$ is a principal ideal of $R$, say $O(y)=R b$, where $b$ is a nonzero element of $R$ and $b$ is not a unit of $R$. Let $x=(1 / b)+R$; then $x$ is an element of $K \subset E(K)$ and $O(x)=R b=O(y)$.

By hypothesis, $R x+R y$ is a direct sum of cyclic $R$-modules. Since $E(K)$ is an indecomposable, injective $R$-module by Lemma $5.4, R x+R y$ must actually be a cyclic $R$-module. Hence there exists an element $z \in E(K)$ such that $R x+R y=R z$. Thus there exist nonzero elements $r, s$ in $R$ such that $x=r z$ and $y=s z$. If $r$ divides $s$, then there exists an element $d \in R$ such that $s=r d$. Then we have $y=s z=d r z=d x$, and so $y$ is an element of $K$, which is a contradiction. Therefore, $s$ divides $r$, and so there exists an element $a \in R$ such that $r=a s$. Hence we have $x=r z=a s z=a y$. By Lemma 3 we then have $O(y)=a O(a y)=a O(x)$. Since $O(y)=R b=O(x)$, it follows that $R b=R a b$. Hence $R a=R$, and so $a$ is a unit in $R$. Therefore, $y=a^{-1} a y=a^{-1} x$ is an element of $K$. This contradiction shows that $E(K)=K$, and so $R$ is an almost maximal valuation ring.

Lemma 5.6. Let $R$ be a local domain. If every finitely generated, torsion $R$-module is a direct sum of cyclic $R$-modules, then $R$ is an almost maximal valuation ring.

( $\left.{ }^{1}\right)$ Also see Theorem 11.1 of Kaplansky's paper Elementary divisors and modules, Trans. Amer. Math. Soc. 66 (1949), 464-491. 
Proof. Let $a, b$ be nonzero elements of $R$. Suppose that $a \notin R b$ and $b \notin R a$. We will show that this yields a contradiction, which will therefore prove that $R$ is a valuation ring. The lemma will then follow from Lemma 5.5.

Let $x=(1 / a)+R$ and $y=(1 / b)+R$, so that $x$ and $y$ are nonzero elements of $K$. Let $A=R x+R y$; then $A$ is a finitely generated torsion $R$-module. Hence by assumption we have $A=A_{1} \oplus \cdots \oplus A_{n}$, where each $A_{i}$ is a cyclic torsion $R$-module. For each $i=1, \ldots, n$ let $p_{i}: A \rightarrow A$ be defined to be the projection on $A_{i}$ with kernel $A_{1} \oplus \cdots \oplus A_{i-1} \oplus A_{i+1} \oplus \cdots \oplus A_{n}$.

Now $A_{i}=p_{i}(A)=p_{i}(R x)+p_{i}(R y)$. But $A_{i}$ is a local $R$-module, and so we must have either $A_{i}=p_{i}(R x)$ or $A_{i}=p_{i}(R y)$. We can assume that $A_{i}=p_{i}(R x)$ for $i \leqq k$ and that $A_{i}=p_{i}(R y)$ for $i \geqq k+1$. Let $B_{1}=A_{1} \oplus \cdots \oplus A_{k}$, and $B_{2}=A_{k+1} \oplus \cdots \oplus A_{n}$, so that $A=B_{1} \oplus B_{2}$. Since $a p_{i}(x)=p_{i}(a x)=p_{i}(0)=0$, we have $p_{i}(x) \subset R x$ for all $i \leqq n$. Therefore $B_{1} \subset R x$. Similarly we have $B_{2} \subset R y$.

Since $A=B_{1} \oplus B_{2}$, there exist $z_{1} \in B_{1}$ and $z_{2} \in B_{2}$ such that $x=z_{1}+z_{2}$. Since $B_{1} \subset R x$ and $B_{2} \subset R y$, there exist elements $r, s$ in $R$ such that $z_{1}=r x$ and $z_{2}=s y$. Hence we have $(1 / a)+R=[(r / a)+R]+[(s / b)+R]=[(r / a)+(s / b)]+R$. Thus there exists an element $c$ in $R$ such that $1 / a=(r b+s a) / a b+c$. Hence $b=r b+s a+c a b$, and so $(1-r) b=(s+c b) a$. If $r$ is not a unit in $R$, then $1-r$ is a unit in $R$, and we would have $b \in R a$ by the preceding equation. This is a contradiction to our assumptions, and so $r$ must be a unit in $R$. Therefore, $x=r^{-1} r x=r^{-1} z_{1} \in B_{1}$. It follows that $B_{1}=R x$. Similarly we have $B_{2}=R y$.

Thus we have $A=B_{1} \oplus B_{2}=R x \oplus R y$, and so $R x \cap R y=0$. Let $u=1 / a$ and $v=1 / b$; then $R x=R u / R$ and $R y=R v / R$. Hence $0=R u / R \cap R v / R=(R u \cap R v) / R$, and so $R u \cap R v=R$. Now we have $(R a+R b)^{-1}=R u \cap R v$. Thus we have shown that if $a, b$ are elements of $R$ such that $a \notin R b$ and $b \notin R a$, then $(R a+R b)^{-1}=R$.

Let $t$ be a nonzero element of $R$ and let $d=b t$ and $e=a t$. If $d \in R e$, then $b \in R a$, and so we have $d \notin R e$. Similarly we have $e \notin R d$. Hence by what we have shown above we have $(R d+R e)^{-1}=R$. Let $D=R u+R v$, and $s=a b t$. Then $s D=R d+R e$, and so $(s D)^{-1}=R$. But $(s D)^{-1}=(1 / s) D^{-1}$, and so we have $D^{-1}=s R$.

If we take $t=1$, then $s=a b$, and we have $D^{-1}=a b R$. If we take $t=a$, then $s=a^{2} b$, and we have $D^{-1}=a^{2} b R$. Thus we have $a b R=a^{2} b R$, which implies that $R=R a$. Therefore, $a$ is a unit in $R$, and so $b \in R a$. This contradiction shows that if $a$ and $b$ are nonzero elements of $R$, then either $a \in R b$, or $b \in R a$. This shows that $R$ is a valuation ring, and hence by Lemma 5.5 an almost maximal valuation ring. This completes the proof of the lemma.

The following theorem generalizes and proves the converse of [3, Theorem 14].

THeOREM 5.7. Let $R$ be an h-local domain. Then the following statements are equivalent:

(1) $R$ is a Prufer domain and $K$ is injective.

(2) $R_{M}$ is an almost maximal valuation ring for every maximal ideal $M$ of $R$.

(3) Every finitely generated torsion $R$-module is a direct sum of cyclic $R$-modules. 
Proof. $(1) \Rightarrow(2)$. Since $R$ is a Prufer ring, $R_{M}$ is a valuation ring for every maximal ideal $M$ of $R$. By Theorem 3.3 we have $0=i n j . \operatorname{dim}_{\cdot_{R}} K=\sup _{M} \operatorname{inj.} \operatorname{dim}_{\cdot_{R_{M}}} K_{M}$. Thus $K_{M}$ is $R_{M}$-injective, and so $R_{M}$ is a maximal valuation ring for every maximal ideal $M$ of $R$.

(2) $\Rightarrow(3)$. Let $T$ be a finitely generated, torsion $R$-module. Then by Theorem 3.1 there exists a finite number of maximal ideals $M_{1}, \ldots, M_{n}$ of $R$ such that $T \cong$ $T_{M_{1}} \oplus \cdots \oplus T_{M_{n}}$. By Lemma 2.1 the $R$-structure and the $R_{M_{i}}$-structure of $T_{M_{i}}$ are the same, and so without loss of generality we may assume that $R$ is a local ring. Then by assumption $R$ is an almost maximal valuation ring. But then by Lemma $5.5, T$ is a direct sum of cyclic $R$-modules.

(3) $\Rightarrow(1)$. Let $M$ be a maximal ideal of $R$, and let $T$ be a finitely generated, torsion $R_{M}$-module. Then by Lemma $2.1, T$ is a finitely generated, torsion $R$-module. Hence by assumption $T$ is a direct sum of cyclic $R$-modules. But by Lemma 2.1 again, $T$ is a direct sum of cyclic $R_{M}$-modules. Thus Lemma 5.6 applies to $R_{M}$, and $R_{M}$ is an almost maximal valuation ring. Since this is true for all maximal ideals $M$ of $R, R$ is a Prufer domain; and by Theorem 3.3 we have

$$
\text { inj. } \underset{R}{\operatorname{dim}} . K=\sup _{M} \text { inj. } \operatorname{dim}_{R_{M}} . K_{M}=0 \text {. }
$$

6. Decomposable torsion-free modules. There is very little that is known about the theory of torsion-free modules over an integral domain. A beginning step in the program is to attempt to find out when a torsion-free module of finite rank is indecomposable. I. Kaplansky has proved that over a maximal valuation ring every torsion-free module of countable rank is a direct sum of modules of rank one ([3, Theorem 12]). This section is concerned with determining the class of all integral domains that have the property that every torsion-free module of finite rank is a direct sum of modules of rank one. In the course of this investigation we exhibit a number of different ways of constructing indecomposable torsion-free modules of rank two for various kinds of integral domains. This considerably sharpens the results in [7, Proposition 7 and Corollary].

Most of this section is devoted to proving the following theorem:

THEOREM 6.1. Let $R$ be an integral domain. Then the following statements are equivalent:

(1) Every torsion-free $R$-module of finite rank is a direct sum of $R$-modules of rank one.

(2) $R$ is an h-local domain with at most two maximal ideals $M_{1}, M_{2}$ and $R_{M_{1}}$, $R_{M_{2}}$ are maximal valuation rings.

Definition. We will say that an integral domain has property $D$, if it satisfies condition (1) of Theorem 6.1. 
LeMma 6.2. Let $R$ be an integral domain with property $D$, and let $S$ be a subring of $Q$ containing $R$. Then $S$ also has property $D$.

Proof. Let $A$ be a torsion-free $S$-module. Suppose that we have $A=A_{1} \oplus A_{2}$, where $A_{1}, A_{2}$ are $R$-submodules of $A$. Let $s$ be a nonzero element of $S$, and $x$ a nonzero element of $A_{1}$. Then $s x=y_{1}+y_{2}$, where $y_{i} \in A_{i}$ for $i=1,2$. Now there exist elements $a$ and $b$ in $R$ such that $b \neq 0$ and $s=a / b$. Then $a x=b s x=b y_{1}+b y_{2}$. But $a x \in A_{1}$, and so $b y_{2}=0$. Hence $y_{2}=0$, and so $s x=y_{1} \in A_{1}$. Therefore, $A_{1}$ is an $S$-module. Similarly $A_{2}$ is an $S$-module. Thus if $R$ has property $D$, so has $S$.

LEMMA 6.3. Let $R$ be an integral domain, complete in the $R$-topology; and let $J$ be a nonzero ideal of $R$. Then $K \otimes_{R} J$ has no proper, $h$-divisible $R$-submodules.

Proof. We have $K \otimes_{R} J \cong Q / J$. Let $B$ be a proper $R$-submodule of $Q$ containing $J$. Since $R$ is complete in the $R$-topology, $R$ is cotorsion as an $R$-module. Therefore $J$ is also a cotorsion $R$-module, and we have $\operatorname{Ext}_{R}^{1}(Q, J)=0$. Since $B$ is a proper $R$-submodule of $Q$, we have $\operatorname{Hom}_{R}(Q, B)=0$. Thus we have an exact sequence:

$$
0=\operatorname{Hom}_{R}(Q, B) \rightarrow \operatorname{Hom}_{R}(Q, B / J) \rightarrow \operatorname{Ext}_{R}^{1}(Q, J)=0,
$$

where the end $R$-modules are zero. Thus $\operatorname{Hom}_{R}(Q, B / J)=0$, and so $B / J$ is $h$ reduced. Therefore, $K \otimes_{R} J$ has no proper, $h$-divisible $R$-submodules.

LemMa 6.4. Let $R$ be an integral domain, complete in the R-topology; and let $I$ and $J$ be nonzero ideals of $R$. Consider an exact sequence:

$$
0 \longrightarrow I \stackrel{i}{\longrightarrow} A \stackrel{p}{\longrightarrow} J \longrightarrow 0,
$$

where $A$ is an $R$-module. Then if $A$ is decomposable, the sequence splits.

Proof. Assume that $A$ has nonzero $R$-submodules $A_{1}$ and $A_{2}$ such that $A=$ $A_{1} \oplus A_{2}$. Suppose the sequence does not split. Let $p_{i}$ be the restriction of $p$ to $A_{i}$ for $i=1,2$. Now $p_{1}$ is a monomorphism. For suppose that $\operatorname{Ker} p_{1} \neq 0$. Since $A_{1}$ and $J$ are torsion-free $R$-modules of rank one, this means that $p_{1}=0$. Hence we have: $\operatorname{Ker} p+A_{2}=A$. If $A_{2} \cap \operatorname{Ker} p \neq 0$, then since $A_{2}$ and $J$ are torsion-free $R$ modules of rank one, we have $A_{2} \subset \operatorname{Ker} p$. This is clearly a contradiction, and so we have $A_{2} \cap \operatorname{Ker} p=0$. Therefore, $A=\operatorname{Ker} p \oplus A_{2}$; but this means the exact sequence splits, which contradicts our assumption. Thus we have $\operatorname{Ker} p_{1}=0$, and so $p_{1}$ is a monomorphism. Similarly $p_{2}$ is a monomorphism. 
Let $J_{1}=p_{1}\left(A_{1}\right)$ and $J_{2}=p_{2}\left(A_{2}\right)$, so that $J=J_{1}+J_{2}$. Define $f: A \rightarrow J_{1} \oplus J_{2}$ by $f\left(x_{1}+x_{2}\right)=\left(p_{1}\left(x_{1}\right), p_{2}\left(x_{2}\right)\right)$, where $x_{i} \in A_{i}$ for $i=1,2$. Since $p_{i}$ is an isomorphism of $A_{i}$ onto $J_{i}$ for $i=1,2$, it follows that $f$ is an isomorphism. Define

$$
\Delta: J_{1} \cap J_{2} \rightarrow J_{1} \oplus J_{2}
$$

by $\Delta(y)=(y,-y)$ for $y \in J_{1} \cap J_{2}$; and define $\gamma: J_{1} \oplus J_{2} \rightarrow J$ by $\gamma\left(\left(y_{1}, y_{2}\right)\right)=y_{1}+y_{2}$, where $y_{i} \in J_{i}$ for $i=1,2$. We have that $\Delta$ is a monomorphism, $\gamma$ is an epimorphism and $\operatorname{Ker} \gamma=\operatorname{Im} \Delta$. Since $\gamma f=p, f i$ maps $I$ into $\operatorname{Im} \Delta$. Thus we can define a map $g: I \rightarrow J_{1} \cap J_{2}$ by $g=\Delta^{-1} f i$. Then $\Delta g=f i$, and $g$ is an isomorphism. Then we have a commutative diagram with exact rows:

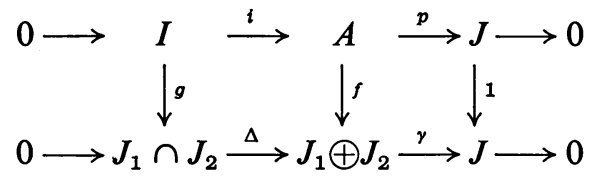

and the vertical maps are isomorphisms. Since the top row does not split, the bottom row does not split either.

Now we have an isomorphism $\mu: K \otimes_{R}\left(J_{1} \oplus J_{2}\right) \rightarrow\left(K \otimes_{R} J_{1}\right) \oplus\left(K \otimes_{R} J_{2}\right)$ defined by $\mu\left(k \otimes\left(x_{1}, x_{2}\right)\right)=\left(k \otimes x_{1}, k \otimes x_{2}\right)$, where $k \in K$ and $x_{i} \in J_{i}$ for $i=1$, 2. Define $\xi: K \otimes_{R}\left(J_{1} \cap J_{2}\right) \rightarrow\left(K \otimes_{R} J_{1}\right) \oplus\left(K \otimes_{R} J_{2}\right)$ by $\xi=\mu(1 \otimes \Delta)$ and define

$$
\eta:\left(K \otimes_{R} J_{1}\right) \oplus\left(K \otimes_{R} J_{2}\right) \rightarrow K \otimes_{R} J
$$

by $\eta=(1 \otimes \gamma) \mu^{-1}$. Then we have a commutative diagram with exact rows:

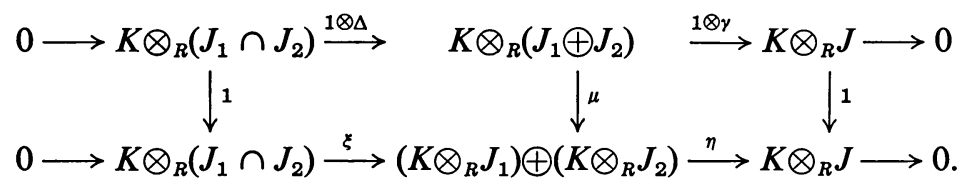

Hence $K \otimes_{R} J=\eta\left(K \otimes_{R} J_{1}\right)+\eta\left(K \bigotimes_{R} J_{2}\right)$. By Lemma 6.3, $K \bigotimes_{R} J$ has no proper $h$-divisible $R$-submodules, and thus we can assume that $\eta\left(K \otimes_{R} J_{1}\right)=K \otimes_{R} J$. Therefore, $\left(K \otimes_{R} J_{1}\right) \oplus\left(K \otimes_{R} J_{2}\right)=\operatorname{Im} \xi+\left(K \otimes_{R} J_{1}\right)$. Now every element of

$$
K \otimes_{R}\left(J_{1} \cap J_{2}\right)
$$

is of the form $k \otimes x$, where $k \in K$ and $x \in J_{1} \cap J_{2}$. We have $\xi(k \otimes x)=(k \otimes x,-k \otimes x)$, and thus if $\xi(k \otimes x) \in K \otimes_{R} J_{1}$, then there exist elements $k_{1} \in K$ and $x_{1} \in J_{1}$ such that $(k \otimes x,-k \otimes x)=\left(k_{1} \otimes x_{1}, 0\right)$. Hence $k \otimes x=0$, and so we have $\operatorname{Im} \xi \cap\left(K \otimes_{R} J_{1}\right)=0$. Thus the bottom row of the above commutative diagram splits. It follows that the 
top row of the above diagram splits. Therefore the following sequence is exact and splits:

$0 \longrightarrow \operatorname{Hom}_{R}\left(K, K \otimes\left(J_{1} \cap J_{2}\right)\right) \stackrel{(1 \otimes \Delta) .}{\longrightarrow} \operatorname{Hom}_{R}\left(K, K \otimes\left(J_{1} \oplus J_{2}\right)\right)$

$$
\stackrel{(1 \otimes \gamma) .}{\longrightarrow} \operatorname{Hom}_{R}(K, K \otimes J) \longrightarrow 0 .
$$

Now by [8, Corollary 3.3] we have a commutative diagram:

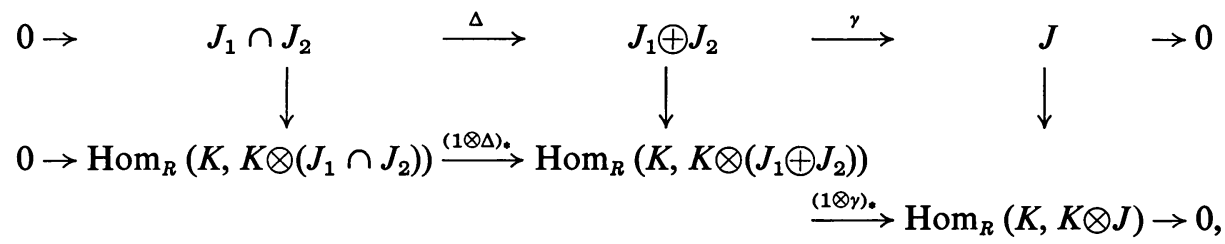

where the bottom row is exact and splits. Since $J_{1} \cap J_{2}, J_{1} \oplus J_{2}$, and $J$ are all torsion-free, cotorsion $R$-modules, we have by [8, Proposition 3.2] that the vertical maps of this last diagram are isomorphisms. Thus the top row of this last diagram splits, which contradicts the fact that we have proved that it does not split. The only way out of this dilemma is that the exact sequence provided by the lemma must have been split to begin with. This concludes the proof of Lemma 6.4.

Proposition 6.5. Let $R$ be an integral domain, complete in the $R$-topology. If $R$ has property $D$, then $R$ is a maximal valuation ring.

Proof. Let $I$ and $J$ be nonzero ideals of $R$ and consider an exact sequence of the form:

$$
0 \rightarrow I \rightarrow A \rightarrow J \rightarrow 0
$$

where $A$ is an $R$-module. By property $D, A$ is a direct sum of two $R$-modules of rank one. Then by Lemma 6.4 , this exact sequence splits. This means that $\operatorname{Ext}_{R}^{1}(J, I)=0$. Since we have $\operatorname{Ext}_{R}^{2}(R / J, I) \cong \operatorname{Ext}_{R}^{1}(J, I)$, it follows that $\operatorname{Ext}_{R}^{2}(R / J, I)$ $=0$. Therefore, inj. $\operatorname{dim}_{._{R}} I=1$. From the exact sequence:

$$
0 \rightarrow I \rightarrow Q \rightarrow K \otimes_{R} I \rightarrow 0
$$

we can then conclude that $K \otimes_{R} I$ is an injective $R$-module. By Lemma 6.3, $K \otimes_{R} I \cong Q / I$ is an indecomposable injective $R$-module.

Let $a$ and $b$ be nonzero elements of $R$. Let $I=R a \cap R b, L_{1}=R a$, and $L_{2}=R b$. Then $L_{i} / I \subset Q / I$ for $i=1,2$; and we have $\left(L_{1} / I\right) \cap\left(L_{2} / I\right)=0$. Since $Q / I$ is an indecomposable, injective $R$-module, either $L_{1} / I=0$ or $L_{2} / I=0$. Hence either $a \in R b$ or $b \in R a$. Therefore, $R$ is a valuation ring. Since we have shown that $K \cong Q / R$ 
is an injective $R$-module, $R$ is an almost maximal valuation ring. But $R$ is complete in the $R$-topology, and so $R$ is a maximal valuation ring.

LEMMA 6.6. Let $R$ be a local domain and consider an exact sequence of the form:

$$
0 \longrightarrow R \stackrel{i}{\longrightarrow} A \stackrel{p}{\longrightarrow} Q \longrightarrow 0
$$

Then if $A$ is a decomposable $R$-module, the exact sequence splits.

Proof. Suppose that $A=A_{1} \oplus A_{2}$, where $A_{1}, A_{2}$ are nonzero $R$-submodules of $A$. If we tensor the exact sequence with $K$ we obtain the isomorphisms: $K \cong K \otimes_{R} R \cong K \otimes_{R} A \cong\left(K \otimes_{R} A_{1}\right) \oplus\left(K \otimes_{R} A_{2}\right)$. But by Corollary $4.2, K$ is an indecomposable $R$-module. Therefore we can assume that $K \otimes_{R} A_{2}=0$, Since $A_{2}$ is torsion-free of rank one, we must then have that $A_{2} \cong Q$.

If $A_{2} \cap \operatorname{Ker} p \neq 0$, then we must have $A_{2} \subset \operatorname{Ker} p=\operatorname{Im} i \cong R$. But since $R$ is $h$-reduced, this can not happen, and so $A_{2} \cap \operatorname{Ker} p=0$. Since $Q$ has no proper divisible $R$-submodules, we have $p\left(A_{2}\right)=Q$. Therefore, $A=A_{2}+\operatorname{Ker} p$, and so $A=A_{2} \oplus \operatorname{Ker} p$. Thus the exact sequence splits.

CoROllary 6.7. Let $R$ be a local domain with property $D$. Then $R$ is a maximal valution ring.

Proof. Consider an exact sequence of the form:

$$
0 \rightarrow R \rightarrow A \rightarrow Q \rightarrow 0
$$

By property $D, A$ is decomposable, and so by Lemma 6.6 the exact sequence splits. Therefore, we have $\operatorname{Ext}_{R}^{1}(Q, R)=0$; and so by [8, Proposition 5.2], $R$ is complete in the $R$-topology. Hence by Proposition $6.5, R$ is a maximal valuation ring.

LEMMA 6.8. Let $R$ be an integral domain with exactly two maximal ideals. Then if $R$ has property $D$, it is an h-local domain.

Proof. Let $M_{1}, M_{2}$ be the maximal ideals of $R$ and let $S=R_{M_{1}}+R_{M_{2}} \subset Q$. Since $R_{M_{1}} \cap R_{M_{2}}=R$, we have an exact sequence:

$$
0 \rightarrow R \rightarrow R_{M_{1}} \oplus R_{M_{2}} \rightarrow S \rightarrow 0 .
$$

By Lemma $6.2, R_{M_{1}}$ has property $D$. Since $R_{M_{1}}$ is a local ring, we can conclude from Corollary 6.7 that $R_{M_{1}}$ is a maximal valuation ring, and hence is a cotorsion $R_{M_{1}}$-module. Since we have $\operatorname{Ext}_{R}^{1}\left(Q, R_{M_{1}}\right) \cong \operatorname{Ext}_{R_{M_{1}}}^{1}\left(Q, R_{M_{1}}\right)$ by [2, Chapter VI, Proposition 4.1.3], it follows that $R_{M_{1}}$ is a cotorsion $R$-module. Similarly $R_{M_{2}}$ is a 
cotorsion $R$-module, and thus so is $R_{M_{1}} \oplus R_{M_{2}}$. Therefore, since $S$ is torsion-free, we have an imbedding: $R \subset H \subset R_{M_{1}} \oplus R_{M_{2}}$ by [8, Proposition 2.8], where $H$ is the completion of $R$ in the $R$-topology. If $R$ were complete, then it would be a local domain by Proposition 6.5. Hence $R$ is not complete, and so $R \neq H$. Thus $H / R$ is not zero; by [8, Proposition 5.2] it is torsion-free and divisible. Since $H / R \subset S$, and since $S$ is torsion-free of rank one, we have $H / R=S$. Therefore, $H=R_{M_{1}} \oplus R_{M_{2}}$. Since $R_{M_{i}}=H\left(M_{i}\right)$ for $i=1,2$, it follows from [8, Theorem 8.5] that $R$ is an $h$-local domain.

Proposition 6.9. Let $R$ be an h-local domain with more than two maximal ideals. Then $R$ has an indecomposable, torsion-free $R$-module of rank two.

Proof. Let $M_{1}, M_{2}, M_{3}$ be three distinct maximal ideals of $R$. Let $V$ be a two dimensional vector space over $Q$ with basis $u$ and $v$. Choose an element $b \in M_{3}$ such that $b \notin M_{1}$ and $b \notin M_{2}$. Let $z=(1 / b) u+v \in Q$. We define three $R$-submodules $C_{1}, C_{2}$, and $C_{3}$ of $V$ as follows. Let $C_{1}=Q u \oplus R_{M_{1}} v ; C_{2}=R_{M_{2}} u \oplus Q v$; and $C_{3}=$ $R_{M_{3}} u \oplus R_{M_{3}} z$. We let $C=C_{1} \cap C_{2} \cap C_{3}$; we will show that $C$ is an indecomposable, torsion-free $R$-module of rank two.

(1) We have $u, z \in C$; and $\left(R_{M_{2}} \cap R_{M_{3}}\right) u \subset C$. Clearly $\left(R_{M_{2}} \cap R_{M_{3}}\right) u \subset C$, and so $u \in C$. Certainly $z \in C_{1}$ and $z \in C_{3}$. Since $b \notin M_{2}$, we have $1 / b \in R_{M_{2}}$; and so $z \in C_{2}$. Therefore $z$ is in the intersection of the $C_{i}$ 's, which is $C$.

(2) We have $C_{1}=C_{M_{1}}$. Since $\left(R_{M_{2}} \cap R_{M_{3}}\right) u \subset C$, we have $R_{M_{1}}\left(R_{M_{2}} \cap R_{M_{3}}\right) u \subset C_{M_{1}}$. But $R_{M_{1}}\left(R_{M_{2}} \cap R_{M_{3}}\right)=Q$, since $R$ is an $h$-local domain. Therefore, $Q u \subset C_{M_{1}}$. In particular, $(1 / b) u \in C_{M_{1}}$. Now $z \in C \subset C_{M_{1}}$, and so $v=z-(1 / b) u \in C_{M_{1}}$. Thus $C_{1}=Q u \oplus R_{M_{1}} v \subset C_{M_{1}}$. But $C \subset C_{1}$, and so $C_{M_{1}} \subset\left(C_{1}\right)_{M_{1}}=C_{1}$. Thus we have $C_{1}=C_{M_{1}}$.

(3) We have $C_{2}=C_{M_{2}}$. Since $u \in C$, we have $R_{M_{2}} u \subset C_{M_{2}}$. Hence $(1 / b) u \in C_{M_{2}}$. Thus $v=z-(1 / b) u \in C_{M_{2}}$. Now $\left(R_{M_{3}} \cap R_{M_{1}}\right) b v \subset C$. For let $a \in R_{M_{3}}$; then $a b v=$ $-a u+a b z \in C_{3}$, and so $R_{M_{3}} b v \subset C_{3}$. Since $Q v \subset C_{2}$, and $R_{M_{1}} v \subset C_{1}$, it follows that $\left(R_{M_{3}} \cap R_{M_{1}}\right) b v \subset C$. Therefore, $R_{M_{2}}\left(R_{M_{3}} \cap R_{M_{1}}\right) b v \subset C_{M_{2}}$. But we have

$$
R_{M_{2}}\left(R_{M_{3}} \cap R_{M_{1}}\right) b=Q b=Q
$$

since $R$ is $h$-local. Hence $Q v \subset C_{M_{2}}$, and so $C_{2}=R_{M_{2}} u \oplus Q v \subset C_{M_{2}}$. Since $C \subset C_{2}$, we have $C_{M_{2}} \subset\left(C_{2}\right)_{M_{2}}=C_{2}$. Thus $C_{2}=C_{M_{2}}$.

(4) We have $C_{3}=C_{M_{3}}$. Since $u, z$ are in $C$, we have $C_{3}=R_{M_{3}} u \oplus R_{M_{3}} z \subset C_{M_{3}}$. On the other hand, since $C \subset C_{3}$, we have $C_{M_{3}} \subset\left(C_{3}\right)_{M_{3}}=C_{3}$. Thus $C_{3}=C_{M_{3}}$.

Now $C$ is a torsion-free $R$-module of rank two, since it contains $u$ and $z$. Suppose that $C$ is decomposable. We then have $C=D_{1} \oplus D_{2}$, where $D_{1}, D_{2}$ are $R$-submodules of $C$ of rank one.

(5) We can assume that $\left(D_{1}\right)_{M_{1}}=Q u$ and $\left(D_{2}\right)_{M_{2}}=Q v$. For we have $C_{1}=C_{M_{1}}=$ $\left(D_{1}\right)_{M_{1}} \oplus\left(D_{2}\right)_{M_{1}}$. If both $\left(D_{1}\right)_{M_{1}}$ and $\left(D_{2}\right)_{M_{1}}$ are reduced, then $C_{1}$ is reduced. But 
$Q u \subset C_{1}$, and so $C_{1}$ is not reduced. Thus we can assume that $\left(D_{1}\right)_{M_{1}}$ is not reduced. Therefore, $\left(D_{1}\right)_{M_{1}}$ is isomorphic to $Q$. Since $Q u$ is the divisible submodule of $C_{1}$, we have $\left(D_{1}\right)_{M_{1}} \subset Q u$. But then we must have $\left(D_{1}\right)_{M_{1}}=Q u$, since both modules have rank one. A similar argument for $C_{2}$ shows that we must have either $\left(D_{1}\right)_{M_{2}}=Q v$, or $\left(D_{2}\right)_{M_{2}}=Q v$. Since $\left(D_{1}\right)_{M_{1}}=Q u$, and $D_{1}$ has rank one, we can not have $\left(D_{1}\right)_{M_{2}}=Q v$. Thus $\left(D_{2}\right)_{M_{2}}=Q v$.

It follows from (5) that we have $D_{1} \subset Q u$ and $D_{2} \subset Q v$. Let $E_{1}=\left(D_{1}\right)_{M_{3}}$ and $E_{2}=\left(D_{2}\right)_{M_{3}}$. Then $E_{1} \subset Q u, E_{2} \subset Q v$, and $C_{3}=C_{M_{3}}=E_{1} \oplus E_{2}$. Now $C_{3}$ is a free $R_{M_{3}}$-module of rank two, and so $E_{1}$ is a free $R_{M_{3}}$-module with generator $x_{1}$, and $E_{2}$ is a free $R_{M_{3}}$-module with generator $x_{2}$.

There exists elements $q_{1}, q_{2}$ in $Q$ such that $x_{1}=q_{1} u$ and $x_{2}=q_{2} v$. Since $z \in C_{3}$, there exist elements $d_{1}, d_{2}$ in $R_{M_{3}}$ such that $(1 / b) u+v=z=d_{1} x_{1}+d_{2} x_{2}=d_{1} q_{1} u+d_{2} q_{2} v$. Thus we have $1=d_{2} q_{2}$. Since $q_{2} v=x_{2} \in C_{3}$, there exist elements $a_{1}, a_{2}$ in $R_{M_{3}}$ such that $q_{2} v=a_{1} u+a_{2} z=\left(a_{1}+a_{2} / b\right) u+a_{2} v$. Thus we have $a_{1}+a_{2} / b=0$ and $a_{2}=q_{2}$. Combining these results we have $0=b d_{2}\left(a_{1}+a_{2} / b\right)=b d_{2} a_{1}+d_{2} a_{2}=b d_{2} a_{1}+1$. Hence $1 / b=-d_{2} a_{1} \in R_{M_{3}}$. But $b \in M_{3}$, and so $1=(1 / b) b \in R_{M_{3}} M_{3}$. But this is contrary to the fact that $R_{M_{3}} M_{3}$ is a proper ideal of $R_{M_{3}}$. This contradiction proves that $C$ is an indecomposable, torsion-free $R$-module of rank two.

Lemma 6.10. Let $R$ be an h-local domain with exactly two maximal ideals $M_{1}$ and $M_{2}$. If $R_{M_{1}}$ and $R_{M_{2}}$ are maximal valuation rings, then $R$ has property $D$.

Proof. Assume that $R_{M_{1}}, R_{M_{2}}$ are maximal valuation rings. Let $A$ be a torsionfree $R$-module of rank one. Then we have inj. $\operatorname{dim}_{\cdot_{M_{i}}} A_{M_{i}} \leqq 1$ for $i=1,2$ by [6, Theorem 4]. Hence by Theorem 3.3 we have that inj. $\operatorname{dim}_{\cdot_{R}} A \leqq 1$. There are only three possibilities for $A$; either $A \cong Q$, or $A$ is isomorphic to an ideal of $R$, or $A$ is a cotorsion $R$-module. For suppose that $A$ is not isomorphic to $Q$, or to an ideal of $R$. We may clearly assume that $A \subset Q$. If $A_{M_{1}}=Q$ and $A_{M_{2}}=Q$, then $A=$ $A_{M_{1}} \cap A_{M_{2}}=Q$, contrary to our assumption. Thus we can assume that $A_{M_{2}} \neq Q$. Then there exists a nonzero element $r \in R$ such that $r A_{M_{2}} \subset R_{M_{2}}$, and so $A_{M_{2}}$ is isomorphic to an ideal of $R_{M_{2}}$. If we have $A_{M_{1}} \neq Q$ also, then there exists a nonzero element $s \in R$ such that $s A_{M_{1}} \subset R_{M_{1}}$. But then we would have $r s \subset r A_{M_{2}} \cap s A_{M_{1}} \subset$ $R_{M_{2}} \cap R_{M_{1}}=R$, and so $A$ would be isomorphic to an ideal of $R$. This contradiction shows that $A_{M_{1}}=Q$. Hence we have $A=A_{M_{1}} \cap A_{M_{2}}=Q \cap A_{M_{2}}=A_{M_{2}}$. Therefore $A$ is isomorphic to an ideal of $R_{M_{2}}$, and so is a cotorsion $R_{M_{2}}$-module. But then by [2, Chapter VI, Proposition 4.1.3] $A$ is also a cotorsion $R$-module.

In the course of the above proof we have seen that $A$ is a cotorsion $R$-module if and only if $A \neq Q$ and either $A=A_{M_{1}}$ or $A=A_{M_{2}}$.

Let $C$ be a torsion-free $R$-module of rank $n<\infty$. We will prove that $C$ is a direct sum of $n R$-modules of rank one by induction on $n$. The case $n=1$ is trivial, and so 
we assume that the assertion is true for torsion-free $R$-modules of rank $<n$, and that $n>1$. We can then clearly assume that $C$ is reduced.

Suppose that $C$ contains a nonzero, pure, cotorsion $R$-submodule $D$ of rank $<n$. Then $C / D$ is a torsion-free $R$-module of rank $<n$, and we have an exact sequence:

$$
\operatorname{Ext}_{R}^{1}\left(Q \otimes_{R} C / D, D\right) \rightarrow \operatorname{Ext}_{R}^{1}(C / D, D) \rightarrow \operatorname{Ext}_{R}^{2}\left(K \otimes_{R} C / D, D\right) .
$$

The first term of this sequence is zero, since $D$ is a cotorsion $R$-module; and the last term is zero, since inj. $\operatorname{dim}_{\cdot_{R}} D=1$. Therefore, $\operatorname{Ext}_{R}^{1}(C / D, D)=0$; and so $C \cong D \oplus C / D$. By the induction hypothesis both $D$ and $C / D$ are direct sums of torsion-free $R$-modules of rank one. Hence $C$ is also such a direct sum. Thus we can assume that $C$ does not contain any nonzero, pure, cotorsion $R$-submodules of rank $<n$.

Let $B$ be a pure $R$-submodule of $C$ of rank $n-1$, and let $A=C / B$. Then $A$ is a torsion-free $R$-module of rank one, and we have an exact sequence:

$$
0 \rightarrow B \rightarrow C \rightarrow A \rightarrow 0 \text {. }
$$

Case I. $A \cong Q$.

Let $V=Q \otimes_{R} C$; then $V$ is the injective envelope of $C$. We can assume that $C_{M_{1}}$ and $C_{M_{2}}$ are contained in $V$. Since $R_{M_{1}}$ is a maximal valuation ring we have $\operatorname{Ext}_{R_{M_{1}}}^{1}\left(Q, B_{M_{1}}\right)=0$. Thus if we tensor exact sequence $\left(^{*}\right)$ with $R_{M_{1}}$ it splits, and we obtain $C_{M_{1}} \cong B_{M_{1}} \oplus Q_{1}$, where $Q_{1}$ is an $R$-submodule of $V$ that is isomorphic to $Q$.

Let $E=Q_{1} \cap C$. Since $V$ is the injective envelope of $C$, we have that $E$ is a nonzero $R$-submodule of $C$, and thus $E$ has rank one. Now $E$ is a pure submodule of $C$. For if $x$ is an element of $C$ and $r$ an element of $R$ such that $r x$ is a nonzero element of $E$, then $r x \in Q_{1}$, and so $x \in Q_{1} \cap C=E$. Thus $C / E$ is a torsion-free $R$-module. Hence $C_{M_{1}} / E_{M_{1}} \cong(C / E)_{M_{1}}$ is a torsion-free $R_{M_{1}}$-module. Since $E_{M_{1}} \subset Q_{1}$, we have $C_{M_{1}} / E_{M_{1}} \cong B_{M_{1}} \oplus Q_{1} / E_{M_{1}}$. If $E_{M_{1}} \neq Q_{1}$, then $Q_{1} / E_{M_{1}}$ is a nonzero torsion $R_{M_{1}}$-module, contrary to the fact that $C_{M_{1}} / E_{M_{1}}$ is torsion-free. Thus we have $E_{M_{1}}=Q_{1}$. Now we also have $E_{M_{2}} \subset Q_{1}$, and so $E=E_{M_{1}} \cap E_{M_{2}}=Q_{1} \cap E_{M_{2}}=E_{M_{2}}$. We can not have $E=Q_{1}$, since $E \subset C$ and $C$ is reduced. Thus by our earlier remarks, $E$ is a pure cotorsion $R$-submodule of $C$ of rank one. This contradicts our assumption that $C$ has no such submodules, and so in the presence of this condition Case I can not arise.

Case II. $A$ is isomorphic to an ideal $I$ of $R$.

We have $\operatorname{Ext}_{R}^{1}(I, B) \cong \operatorname{Ext}_{R}^{2}(R / I, B)$. But inj. $\operatorname{dim}_{\cdot_{R}} B=1$, and so $\operatorname{Ext}_{R}^{2}(R / I, B)=0$. Therefore, $\operatorname{Ext}_{R}^{1}(I, B)=0$, and so exact sequence $\left(^{*}\right)$ splits. Thus we have $C \cong B \oplus I$, and by the induction hypothesis $C$ is a direct sum of $R$-modules of rank one.

If $\operatorname{Hom}_{R}(C, R) \neq 0$, then there exists an exact sequence of the form (*) with $A$ isomorphic to a nonzero ideal $I$ of $R$. Thus by Case II we can assume without loss of generality that $\operatorname{Hom}_{R}(C, R)=0$. 
Case III. $A$ is a cotorsion $R$-module.

By our earlier remarks we can assume that $A=A_{M_{2}}$ and $A \nsubseteq Q$. Now by the induction hypothesis we have $B=B_{1} \oplus \cdots \oplus B_{n-1}$, where each $B_{i}$ is an $R$-submodule of $B$ of rank one. Since $B_{1}$ is pure in $B$ and $B$ is pure in $C$, it follows that $B_{1}$ is pure in $C$. By our assumptions $B_{1}$ is not a cotorsion $R$-module, and $B_{1} \not Q$. Therefore, $B_{1}$ is isomorphic to an ideal of $R$. Hence $\operatorname{Hom}_{R}\left(B_{1}, R\right) \neq 0$. Since $\operatorname{Hom}_{R}\left(B_{1}, R\right) \subset \operatorname{Hom}_{R}(B, R)$, we have that $\operatorname{Hom}_{R}(B, R) \neq 0$. Thus $\operatorname{Hom}_{R}(B, R)$ is a nonzero, torsion-free $R$-module. Now we have an exact sequence:

$$
0=\operatorname{Hom}_{R}(C, R) \rightarrow \operatorname{Hom}_{R}(B, R) \rightarrow \operatorname{Ext}_{R}^{1}(A, R) .
$$

Therefore, $\operatorname{Ext}_{R}^{1}(A, R)$ is not a torsion $R$-module.

Since $R$ is an $h$-local domain, we have $R_{M_{1}}+R_{M_{2}}=Q$. Of course $R=R_{M_{1}} \cap R_{M_{2}}$, and thus we have an exact sequence:

$$
0 \rightarrow R \rightarrow R_{M_{1}} \oplus R_{M_{2}} \rightarrow Q \rightarrow 0 .
$$

Now

$$
\operatorname{Hom}_{R}\left(A, R_{M_{1}}\right)=\operatorname{Hom}_{R}\left(A_{M_{2}}, R_{M_{1}}\right)=0
$$

by [8, Lemma 8.3]. Since $\operatorname{Hom}_{R}(A, R) \subset \operatorname{Hom}_{R}\left(A, R_{M_{1}}\right)$, we have $\operatorname{Hom}_{R}(A, R)=0$ also. Thus from the last exact sequence we obtain an exact sequence:

$$
0 \rightarrow \operatorname{Hom}_{R}\left(A, R_{M_{2}}\right) \rightarrow \operatorname{Hom}_{R}(A, Q) \rightarrow \operatorname{Ext}_{R}^{1}(A, R) \rightarrow \operatorname{Ext}_{R}^{1}\left(A, R_{M_{1}} \oplus R_{M_{2}}\right) .
$$

Since rank $A=1$, we have $\operatorname{Hom}_{R}(A, Q) \cong Q$. Now $R_{M_{1}} \oplus R_{M_{2}}$ is a cotorsion $R$ module and has injective dimension one over $R$. Thus $\operatorname{Ext}_{R}^{1}\left(A, R_{M_{1}} \oplus R_{M_{2}}\right)=0$. Hence the last exact sequence reduces to the following exact sequence:

$$
0 \rightarrow \operatorname{Hom}_{R}\left(A, R_{M_{2}}\right) \rightarrow Q \rightarrow \operatorname{Ext}_{R}^{1}(A, R) \rightarrow 0 .
$$

Since $A \cong A_{M_{2}}$ is isomorphic to an ideal of $R_{M_{2}}$, we have that $\operatorname{Hom}_{R}\left(A, R_{M_{2}}\right)$ is not zero. Therefore, the above exact sequence shows that $\operatorname{Ext}_{R}^{1}(A, R)$ is a torsion $R$ module. But we have shown that $\operatorname{Ext}_{R}^{1}(A, R)$ contains a nonzero, torsion-free $R$-module. This contradiction shows that Case III can not arise.

Having now considered all possible cases, we have completed the proof of the lemma.

Proof of Theorem 6.1. Assume that $R$ has property $D$. If $R$ has only one maximal ideal, then $R$ is a maximal valuation ring by Corollary 6.7. Hence we may assume that $R$ has more than one maximal ideal. Let $M_{1}, M_{2}$ be two different maximal ideals of $R$, and let $S$ be the complement in $R$ of $M_{1} \cup M_{2}$. Then $R_{S}$ has only two maximal ideals; and by Lemma $6.2 R_{S}$ has property $D$. Thus by Lemma $6.8, R_{S}$ is an $h$-local domain. This proves that every nonzero prime ideal of $R$ is contained in only one maximal ideal of $R$. Suppose that $R$ has still a third maximal 
ideal $M_{3}$. Let $T$ be the complement of $M_{1} \cup M_{2} \cup M_{3}$ in $R$. Then by what we have just proved $R_{T}$ is an $h$-local domain with only three maximal ideals. $R_{T}$ has property $D$ by Lemma 6.2. But this contradicts Proposition 6.9. Thus $R$ is an $h$-local domain with only two maximal ideals $M_{1}, M_{2}$ and $R_{M_{1}}, R_{M_{2}}$ are maximal valuation rings.

Conversely, if $R$ is a maximal valuation ring then Kaplansky has proved a stronger result than property $D$ (see [3, Theorem 12] or [6, Theorem 9]). If $R$ is an $h$-local domain with exactly two maximal ideals $M_{1}, M_{2}$ and $R_{M_{1}}$ and $R_{M_{2}}$, are maximal valuation rings, then $R$ has property $D$ by Lemma 6.10 .

This completes the proof of Theorem 6.1 .

REMARKS. It is an open question whether or not there can exist an integral domain with two maximal ideals $M_{1}, M_{2}$ such that $R_{M_{1}}, R_{M_{2}}$ are maximal valuation rings. Kaplansky has proved in [3, Theorem 12] that if $R$ is a maximal valuation ring, then every torsion-free $R$-module of countable rank is a direct sum of $R$ modules of rank one. It is a reasonable conjecture that the converse of this is true; namely, if an integral domain has this property, then it must be a maximal valuation ring.

It is of some interest to find as many kinds of integral domains as possible for which the possession of property $D$ will imply that the domain is a maximal valuation ring. The next proposition and its corollary will provide a partial answer to this question.

An immediate corollary of a result that Kaplansky has proved in [4] is that if $R$ is a Noetherian domain with property $D$, then $R$ is a complete, discrete, valuation ring. A trivial modification of his proof will prove the next proposition. We will give the full proof of the proposition for, a priori, it is not clear which portion of Kaplansky's proof we will be using.

Definition. We say that an integral domain is powerful, if $Q$ is a countably generated $R$-module. $R$ is locally powerful, if $R_{M}$ is powerful for every maximal ideal $M$ of $R$.

Proposition 6.11. Let $R$ be a locally powerful integral domain. Then $R$ has property $D$ if and only if it is a maximal valuation ring.

Proof. Assume that $R$ has property $D$. Suppose that $R$ is not a maximal valuation ring. Then by Theorem $6.1, R$ is an $h$-local domain with exactly two maximal ideals $M_{1}, M_{2}$ and $R_{M_{1}}, R_{M_{2}}$ are maximal valuation rings. By assumption $K_{M_{1}}$ is a countably generated $R_{M_{1}}$-module. Since $K_{M_{1}}$ is a torsion $R_{M_{1}}$-module, it follows from Lemma 2.1 that a set of $R_{M_{1}}$ generators of $K_{M_{1}}$ is also a set of $R$ generators of $K_{M_{1}}$. Thus $K_{M_{1}}$ is a countably generated $R$-module. Similarly $K_{M_{2}}$ is a countably generated $R$-module. Since $K=K_{M_{1}} \oplus K_{M_{2}}, K$ is also a countably generated $R$ module. Therefore, $Q$ is a countably generated $R$-module. Thus there exist sequences $\left\{a_{n}\right\}$ and $\left\{b_{n}\right\}$ of elements of $R$ such that $\left\{1 / a_{n}\right\}$ generates $Q$ over $R$ and $a_{n+1}=b_{n+1} a_{n}$. 
Now $R$ has infinitely many units. For if we take $p$ to be an element in $M_{1}$ that is not in $M_{2}$, and $q$ to be an element in $M_{2}$ that is not in $M_{1}$, then $p+q^{i}$ is a unit for each positive integer $i$, and these units are all distinct. Thus there exists a unit $u \in R$ such that $u \neq \pm 1$.

Since $R$ is an $h$-local domain and $R_{M_{1}}, R_{M_{2}}$ are complete in the $R$-topology, we have $H=R_{M_{1}} \oplus R_{M_{2}}$, where $H$ is the completion of $R$. Thus $h=\left(u, u^{-1}\right)$ is an element of $H$. Now the $R$-topology on $R$ is given by the submodules $R a_{n}$. Therefore, we can find a Cauchy sequence $\left\{d_{n}\right\}$ of elements of $R$ such that $d_{n}$ converges to $h$ in $H$, and such that $d_{n}-d_{n+1} \in R a_{n}$.

Let $V$ be a two dimensional vector space over $Q$ with basis $x$ and $y$, and define a sequence of elements $\left\{w_{n}\right\}$ of $V$ by setting $w_{n}=x+d_{n} y$. Let $N$ be the $R$-submodule of $V$ generated by the elements $y, w_{0},\left(1 / a_{1}\right) w_{1}, \ldots,\left(1 / a_{n}\right) w_{n}, \ldots$ Then $N$ is a torsionfree $R$-module of rank two. Since $R$ has property $D, N$ is decomposable; and thus there exists an idempotent endomorphism $\varphi$ of $N$ such that $\varphi(N)$ is of rank one. Modifying $\varphi$ by a scalar, we may assume that $\varphi(x)$ and $\varphi(y)$ are linear combinations of $x$ and $y$ with coefficients in $R$; say $\varphi(x)=r_{1} x+r_{2} y$ and $\varphi(y)=r_{3} x+r_{4} y$, with the elements $r_{i}$ in $R$. The matrix of $\varphi$ has determinant 0 and trace $\neq 0$; i.e.,

$$
r_{1} r_{4}=r_{2} r_{3} \text {, and } r_{1}+r_{4} \neq 0 \text {. }
$$

Since $w_{n}=x+d_{n} y$ is divisible by $a_{n}$ in $N$, so is

$$
\varphi\left(x+d_{n} y\right)-\left(r_{1}+d_{n} r_{3}\right)\left(x+d_{n} y\right)=\left[\left(r_{2}+d_{n} r_{4}\right)-d_{n}\left(r_{1}+d_{n} r_{3}\right)\right] y .
$$

A computation will show (exactly as in [4]) that $Q y \cap N=R y$; and thus we have $\left(r_{2}+d_{n} r_{4}\right)-d_{n}\left(r_{1}+d_{n} r_{3}\right) \equiv 0\left(\bmod R a_{n}\right)$. Passing to the limit in $H$ we obtain:

$$
\left(r_{2}+h r_{4}\right)-h\left(r_{1}+h r_{3}\right)=0 .
$$

Since $h=\left(u, u^{-1}\right)$, equation (3) holds with $h$ replaced by either $u$ or $u^{-1}$.

If $r_{1}=0$, then $r_{4} \neq 0$ and either $r_{2}=0$ or $r_{3}=0$. From this we deduce that $u=u^{-1}$, so that $u^{2}=1$, which is a contradiction. Hence $r_{1} \neq 0$. We then have by (1):

$$
r_{2}+h r_{4}=\left(r_{2} / r_{1}\right)\left(r_{1}+h r_{3}\right)
$$

Comparing (3) and (4) we find that both $u$ and $u^{-1}$ are equal to $r_{2} / r_{1}$ or $-r_{3} / r_{1}$. Since $u \neq u^{-1}$, it must be the case that one is $r_{2} / r_{1}$ and the other $-r_{3} / r_{1}$. But then $r_{2} r_{3}=-r_{1}^{2}$, whence $r_{1}+r_{4}=0$, contradicting (1).

Thus we have that $R$ has only a single maximal ideal, and so is a maximal valuation ring. Of course if $R$ is a maximal valuation ring, then $R$ has property $D$ by Theorem 6.1.

COROllary 6.12. Let $R$ be an integral domain with the descending chain condition on prime ideals. Then $R$ has property $D$ if and only if $R$ is a maximal valuation ring. 
Proof. Assume that $R$ has property $D$. Let $M$ be a maximal ideal of $R$. Then by Lemma $6.2, R_{M}$ also has property $D$, and is a local ring. Therefore by Theorem $6.1, R_{M}$ is a maximal valuation ring. Hence the prime ideals of $R_{M}$ are linearly ordered. Since the prime ideals of $R_{M}$ are in one to one, order preserving, correspondence with the prime ideals of $R$ that are contained in $M, R_{M}$ also has the descending chain condition on prime ideals. Thus $R_{M}$ has only finitely many prime ideals. Let $P$ be the minimal, nonzero prime ideal of $R$, and let $s$ be a nonzero element of $P$. Let $S$ be the multiplicatively closed subset of $R_{M}$ consisting of the powers of $s$. Then every prime ideal of $R_{M}$ meets $S$, and hence $\left(R_{M}\right)_{S}$ has no nonzero prime ideals. Therefore, $\left(R_{M}\right)_{S}$ is a field, and so $\left(R_{M}\right)_{S}=Q$, the quotient field of $R_{M}$. Thus the quotient field of $R_{M}$ is a countably generated $R_{M}$-module. We have thus proved that $R$ is a locally powerful domain. It then follows from Proposition 6.11 that $R$ is a maximal valuation ring. The converse follows from Theorem 6.1.

Corollary 6.13. Let $R$ be a Noetherian domain. Then $R$ can not have two distinct rank one prime ideals $P_{1}, P_{2}$ such that $R_{P_{1}}, R_{P_{2}}$ are complete, discrete, valuation rings.

Proof. Suppose that $R$ has two such prime ideals $P_{1}$ and $P_{2}$. Let $S$ be the complement in $R$ of $P_{1} \cup P_{2}$. Then $R_{S}$ has the same property as $R$. Hence we may assume that $R$ has Krull dimension one, and two maximal ideals $P_{1}$ and $P_{2}$ such that $R_{P_{1}}, R_{P_{2}}$ are complete discrete valuation rings. Then $R$ is an $h$-local domain, and so by Theorem $6.1, R$ has property $D$. But $R$ is locally powerful, and thus we have a contradiction by Proposition 6.11. This proves the corollary.

REMARKS. It is an open question whether a Noetherian domain can have even one rank one prime ideal $P$ such that $R_{P}$ is a complete, discrete, valuation ring without already being a complete, discrete, valuation ring.

7. Indecomposable $h$-divisible modules. Throughout this section, unless otherwise specified, $R$ will be a local, Noetherian domain of Krull dimension one. We will let $M$ denote the maximal ideal of $R$, and $E$ the injective envelope of $R / M$. If $A$ is an $R$-module, we will denote the dual $\operatorname{Hom}_{R}(A, E)$ of $A$ by $A^{*}$. We have a canonical imbedding of $A$ into $A^{* *}$, and thus $A^{*}=0$ if and only if $A=0$.

Let $\hat{R}$ be the completion of $R$ in the $M$-adic topology on $R$. Then $\hat{R}=H$, the completion of $R$ in the $R$-topology. If $I$ is a nonzero ideal of $R$ and $I$ its completion in $\hat{R}$, then $\hat{I}=\hat{R} \otimes_{R} I$ and we have $\hat{R} / \hat{I} \cong R / I$. The $\hat{R}$-injective envelope of $\hat{R} / \hat{M}$ is again $E$; and if $A$ is a torsion $R$-module, then $A$ is an $\hat{R}$-module and $\operatorname{Hom}_{R}(A, E) \cong$ $\operatorname{Hom}_{R}(A, E)=A^{*}$. If $D$ is an $R$-module with D.C.C. (descending chain condition), we have $D \cong \operatorname{Hom}_{\hat{R}}\left(D^{*}, E\right)$ by [5, Corollary 4.3].

If $A$ is an $R$-module, we will let $t(A)$ denote the torsion submodule of $A$. 
LEMMA 7.1. Let $S$ be a torsion-free, reduced $R$-module of rank one (i.e., $S$ is a proper $R$-submodule of $Q)$. Then the following statements are equivalent:

(1) $K \otimes_{R} S$ is an indecomposable $R$-module.

(2) $\left(K \otimes_{R} S\right)^{*}$ is an indecomposable $R$-module.

(3) $t\left(S^{*}\right)$ is an indecomposable $R$-module.

Proof. (1) $\Leftrightarrow(2)$ : By [7, Theorem 1], $K \bigotimes_{R} S$ has D.C.C. Hence we have $K \bigotimes_{R} S \cong$ $\operatorname{Hom}_{\hat{R}}\left(\left(K \otimes_{R} S\right)^{*}, E\right)$. Since $\left(K \otimes_{R} S\right)^{*}$ is a cotorsion $R$-module by [8, Proposition 1.3], its $R$ and $\hat{R}$ direct summands coincide by [8, Proposition 5.6]. Thus $K \otimes_{R} S$ is an indecomposable $R$-module if and only if $\left(K \otimes_{R} S\right)^{*}$ is an indecomposable $R$ module.

(2) $\Leftrightarrow(3)$. By [8, Proposition 1.3], $\left(K \otimes_{R} S\right)^{*}$ is a torsion-free, cotorsion $R$ module. Thus by [8, Corollary 3.3], $\left(K \otimes_{R} S\right)^{*}$ is an indecomposable $R$-module if and only if $K \otimes_{R}\left(K \otimes_{R} S\right)^{*}$ is an indecomposable $R$-module. It only remains to show that $K \otimes_{R}\left(K \otimes_{R} S\right)^{*} \cong t\left(S^{*}\right)$.

Now we have an exact sequence:

$$
0 \rightarrow S \rightarrow Q \rightarrow K \otimes_{R} S \rightarrow 0
$$

and hence an exact sequence:

$$
0 \rightarrow\left(K \otimes_{R} S\right)^{*} \rightarrow Q^{*} \rightarrow S^{*} \rightarrow 0
$$

Tensoring this last sequence with $K$, we obtain the isomorphisms: $\operatorname{Tor}_{1}^{R}\left(K, S^{*}\right) \cong$ $K \otimes_{R}\left(K \otimes_{R} S\right)^{*}$. By [2, Chapter VII, Proposition 2.2] we have that $t\left(S^{*}\right) \cong$ $\operatorname{ior}_{1}^{R}\left(K, S^{*}\right)$. Thus $t\left(S^{*}\right) \cong K \bigotimes_{R}\left(K \otimes_{R} S\right)^{*}$, and the proof of the lemma is complete.

COROLlaRY 7.2. Let I be an ideal of $R$. Then the following statements are equivalent:

(1) $K \otimes_{R} I$ is an indecomposable R-module.

(2) $I$ is an indecomposable $R$-module.

(3) $I^{*}$ is an indecomposable R-module.

Proof. $(1) \Leftrightarrow(2)$. Since $I \cong \hat{R} \otimes_{R} I$, we have by [8, Proposition 7.2] that $I=$ $\operatorname{Ext}_{R}^{1}(K, I)$. Thus the equivalence of (1) and (2) follows from [8, Corollary 3.3].

$(1) \Leftrightarrow(3)$. From the exact sequence: $0 \rightarrow I \rightarrow R$, we obtain the exact sequence: $E=R^{*} \rightarrow I^{*} \rightarrow 0$. Thus $t\left(I^{*}\right)=I^{*}$, and so the equivalence of (1) and (3) follows from Lemma 7.1 .

LEMMA 7.3. Assume that $\hat{R}$ has only two prime ideals. Let $B$ be any finitely generated $R$-submodule of $E$. Then any divisible $R$-submodule of $E / B$ is an indecomposable $R$-module. 
Proof. If $X$ is any $R$-submodule of $E$, define $X^{\prime}$ to be the annihilator of $X$ in $\hat{R}$. Let $J=B^{\prime}$; then by [5, Theorem 4.2] we have that $\hat{R} / J \cong B^{*}$. Since $B^{*}$ has D.C.C. by [5, Corollary 4.3], it follows that $J$ is an $\hat{M}$-primary ideal of $\hat{R}$.

Let $D / B$ be a divisible $R$-submodule of $E / B$, where $D$ is an $R$-submodule of $E$ containing $B$. Suppose that we have $D / B=D_{1} / B \oplus D_{2} / B$, where $D_{1}, D_{2}$ are $R$ submodules of $D$ containing $B$. Then $D_{1}+D_{2}=D$ and $D_{1} \cap D_{2}=B$. By [5, Theorem 4.2] we then have that $\left(D_{1} \cap D_{2}\right)^{\prime}=D_{1}^{\prime}+D_{2}^{\prime}$. Therefore, $J=D_{1}^{\prime}+D_{2}^{\prime}$, and $D_{1}^{\prime}, D_{2}^{\prime}$ are ideals of $\hat{R}$.

Let $P$ be the other prime ideal of $\hat{R}$. Suppose that neither $D_{1}^{\prime}$ nor $D_{2}^{\prime}$ are $\hat{M}$ primary ideals of $\hat{R}$. Then $D_{1}^{\prime} \subset P$ and $D_{2}^{\prime} \subset P$. Hence $J=D_{1}^{\prime}+D_{2}^{\prime} \subset P$. Since $J$ is an $\hat{M}$-primary ideal, this is a contradiction. Thus we can assume that $D_{1}^{\prime}$ is an $\hat{M}$ primary ideal of $\hat{R}$. Therefore, $\hat{R} / D_{1}^{\prime}$ has D.C.C.

Now by [5, Theorem 4.2] we have $D_{1} \cong\left(\hat{R} / D_{1}^{\prime}\right)^{*}$, and by [5, Corollary 4.3] we have that $\left(\hat{R} / D_{1}^{\prime}\right)^{*}$ is a finitely generated $\hat{R}$-module. Since the $\hat{R}$-structure and the $R$-structure of $D_{1}$ are the same, $D_{1}$ is a finitely generated $R$-module. Therefore, $D_{1} / B$ is a finitely generated $R$-module. But $D_{1} / B$ is a direct summand of $D / B$, and hence is a divisible $R$-module. Thus we have $D_{1} / B=0$, and so $D / B$ is an indecomposable $R$-module.

THEOREM 7.4. The following statements are equivalent:

(1) $\hat{R}$ has exactly two prime ideals.

(2) $K \otimes_{R} S$ is an indecomposable $R$-module for every torsion-free $R$-module $S$ of rank one.

(3) $K \otimes_{R} I$ is an indecomposable $R$-module for every ideal I of $R$.

Proof. (1) $\Rightarrow(2)$. Let $S$ be a reduced, torsion-free $R$-module of rank one (i.e., $S$ is a nonzero proper $R$-submodule of $Q$ ). By [7, Theorem 1], $S / M S$ has D.C.C. Since $S / M S$ is annihilated by $M$, it has finite length, and thus is finitely generated. Thus there exists a nonzero, finitely generated $R$-submodule $I$ of $S$ such that $M S+I=S$. Hence we have $M(S / I)=S / I$. If $r$ is any nonzero, nonunit of $R$, there exists an integer $n>0$ such that $M^{n} \subset R r$. Hence $S / I=r(S / I)$, and so $S / I$ is divisible. We can assume without loss of generality that $I$ is a nonzero ideal of $R$.

We have an exact sequence:

$$
0 \rightarrow(S / I)^{*} \rightarrow S^{*} \rightarrow I^{*} \rightarrow 0
$$

Since $S / I$ is divisible, $(S / I)^{*}$ is torsion-free. Thus we have $t\left(S^{*}\right) \subset t\left(I^{*}\right)$. Now as we have seen in the proof of Lemma 7.1, we have $t\left(S^{*}\right) \cong K \otimes_{R}\left(K \otimes_{R} S\right)^{*}$. Therefore $t\left(S^{*}\right)$ is a divisible submodule of $t\left(I^{*}\right)$.

Let $B=(R / I)^{*}$; then we have an exact sequence:

$$
0 \rightarrow B \rightarrow E \rightarrow I^{*} \rightarrow 0
$$


and so $I^{*} \cong E / B$. Thus $t\left(I^{*}\right)=I^{*}$, and so $t\left(S^{*}\right)$ is isomorphic to a divisible submodule of $E / B$. Since $R / I$ has D.C.C., $B$ is a finitely generated $R$-submodule of $E$ by [5, Theorem 4.2]. Therefore, Lemma 7.3 applies and $t\left(S^{*}\right)$ is indecomposable. It follows from Lemma 7.1 that $K \otimes_{R} S$ is indecomposable.

(2) $\Rightarrow(3)$. Trivial.

(3) $\Rightarrow(1)$. Suppose that $\hat{R}$ has more than two prime ideals. Then we have $n>1$ distinct prime ideals $P_{1}, \ldots, P_{n}$ of rank 0 in $\hat{R}$. Thus in $\hat{R}$ we have $0=J \cap L$, an irredundant decomposition of 0 , where $J$ is a $P_{1}$-primary ideal; and $P_{2}, \ldots, P_{n}$ belong to the ideal $L$. Now $J+L$ is an $\hat{M}$-primary ideal of $\hat{R}$. For if $P_{j} \supset J+L$ for some $j, 1 \leqq j \leqq n$, then $P_{j} \supset J$; and so $P_{j}=P_{1}$. But $P_{1} \phi L$, which is a contradiction. Thus $J+L$ is an $\hat{M}$-primary ideal of $\hat{R}$. Let $I=(J+L) \cap R$. Since $J+L$ is $\hat{M}$-primary, we have $\hat{I}=\hat{R} I=J+L$.

If $Y$ is any ideal of $\hat{R}$, let $\operatorname{Ann}(Y, E)=\{x \in E \mid Y x=0\}$. We set $B=\operatorname{Ann}(J, E)$ and $C=\operatorname{Ann}(L, E)$. By [5, Theorem 4.2] we have $B+C=\operatorname{Ann}(J \cap L, E)$ and $B \cap C=\operatorname{Ann}(J+L, E)$. Since $J \cap L=0$ and $J+L=\hat{I}$, we have $B+C=E$ and $B \cap C=\operatorname{Ann}(\hat{I}, E)$.

Let $A=$ Ann $(\hat{I}, E)=B \cap C$. Then we have $E / A=(B+C) / A \cong B / A \oplus C / A$. By [5, Theorem 4.2], $E / A \cong \operatorname{Hom}_{\hat{R}}(\hat{I}, E)$; and so we have $(E / A)^{*} \cong \hat{I}$. Since $\hat{I}$ is an indecomposable $R$-module by Corollary 7.2 , we must have $(B / A)^{*}=0$ or $(C / A)^{*}=0$. Thus $B=A$, or $C=A$. If $B=A$, then $J=\hat{l}$, and so $J \supset L$. Thus $0=J \cap L=L$, which contradicts the irredundancy of the decomposition of 0 . Similarly we can not have $C=A$. This contradiction shows that $\hat{R}$ has exactly two prime ideals.

REMARKS. It is a matter of classical ideal theory that the following conditions are equivalent (see [9]).

(1) $\hat{R}$ has exactly two prime ideals.

(2) Every zero divisor of $\hat{R}$ is nilpotent.

(3) The zero ideal of $\hat{R}$ is a primary ideal.

(4) The integral closure of $R$ is a valuation ring.

(5) Every ring extension of $R$ in $Q$ is a local ring.

Theorem 7.4 provides the solution for a problem raised in [7, p. 573]; namely we have the following corollary.

COROllaRY 7.5. Let $R$ be a Noetherian integral domain. Then every R-homomorphic image of $Q$ is indecomposable if and only if $R$ is a local domain of Krull dimension one and $\hat{R}$ has exactly two prime ideals.

Proof. Suppose that every $R$-homomorphic image of $Q$ is indecomposable. Then by [7, Theorem 2], $R$ is a local domain of Krull dimension one. The corollary now follows directly from Theorem 7.4.

The following theorem can be derived without any difficulty from classical ideal theory (see [9]). We introduce it here mainly because it can now be proved by the 
methods of this section, and because it is intimately related to the theorems of this section. It is of interest to compare this theorem with Theorem 4.5.

THEOREM 7.6. Let $R$ be a Noetherian domain of Krull dimension one. Then $\hat{R}$ is an integral domain if and only if every proper $R$-submodule of $Q$ is finitely generated.

Proof. Assume that $\hat{R}$ is an integral domain. It follows that $R$ is then a local domain. Let $S$ be an $R$-submodule of $Q$ which is not finitely generated. We can assume that $R \subset S$. Since $S / R$ has D.C.C. by [7, Theorem 1], we can further assume that every proper $R$-submodule of $S / R$ is finitely generated. Now $S / R$ is divisible. For, if not, there exists a nonzero element $r \in R$ such that $r(S / R) \neq S / R$. Thus $r S+R$ is finitely generated, from which it follows that $S$ is finitely generated. This contradiction shows that $S / R$ is divisible.

Let $E$ be the injective envelope of $R / M$, where $M$ is the maximal ideal of $R$. Since $(S / R)^{*} \neq 0$, and since $E$ has no proper divisible submodule by [7, Proposition 2], it follows that there exists an epimorphism of $S / R$ onto $E$. Let $A / R$ be the kernel of this epimorphism, where $A$ is an $R$-submodule of $E$. Then we have an exact sequence:

$$
0 \rightarrow A \rightarrow S \rightarrow E \rightarrow 0
$$

Tensoring this exact sequence with $K$ we obtain an exact sequence:

$$
0 \rightarrow \operatorname{Tor}_{1}^{R}(K, E) \rightarrow K \otimes_{R} A \rightarrow K \otimes_{R} S \rightarrow 0 .
$$

Since $\operatorname{Tor}_{1}^{R}(K, E) \cong E$, this sequence yields the isomorphism: $K \otimes_{R} A \cong E \oplus K \otimes_{R} S$. But $K \otimes_{R} A$ is indecomposable by Theorem 7.4, and so $K \otimes_{R} S=0$. This means that $S=Q$. Therefore we have proved that every proper $R$-submodule of $Q$ is finitely generated.

Conversely, if every proper $R$-submodule of $Q$ is finitely generated, then $\hat{R}$ is an integral domain by Theorem 4.5 .

\section{BIBLIOGRAPHY}

1. H. Bass, Projective modules over algebras, Ann. of Math. 73 (1961), 532-542.

2. H. Cartan and S. Eilenberg, Homological algebra, Princeton Univ. Press, Princeton, N. J., 1956.

3. I. Kaplansky, Modules over Dedekind rings and valuation rings, Trans. Amer. Math. Soc. 72 (1952), 327-340.

4. - —, Decomposability of modules, Proc. Amer. Math. Soc. 13 (1962), 532-535.

5. E. Matlis, Injective modules over Noetherian rings, Pacific J. Math. 8 (1958), 511-528.

6. - - Injective modules over Prufer rings, Nagoya Math. J. 15 (1959), 57-69.

7. - Some properties of Noetherian domains of dimension 1, Canad. J. Math. 13 (1961), 569-586.

8. - Cotorsion modules, Mem. Amer. Math. Soc. No. 49, (1964).

9. D. G. Northcott, General theory of one dimensional local rings, Proc. Glasgow Math. Assoc. 2 (1956), 159-169.

\section{NORTHWESTERN UNIVERSITY,} Evanston, ILlinoIs 